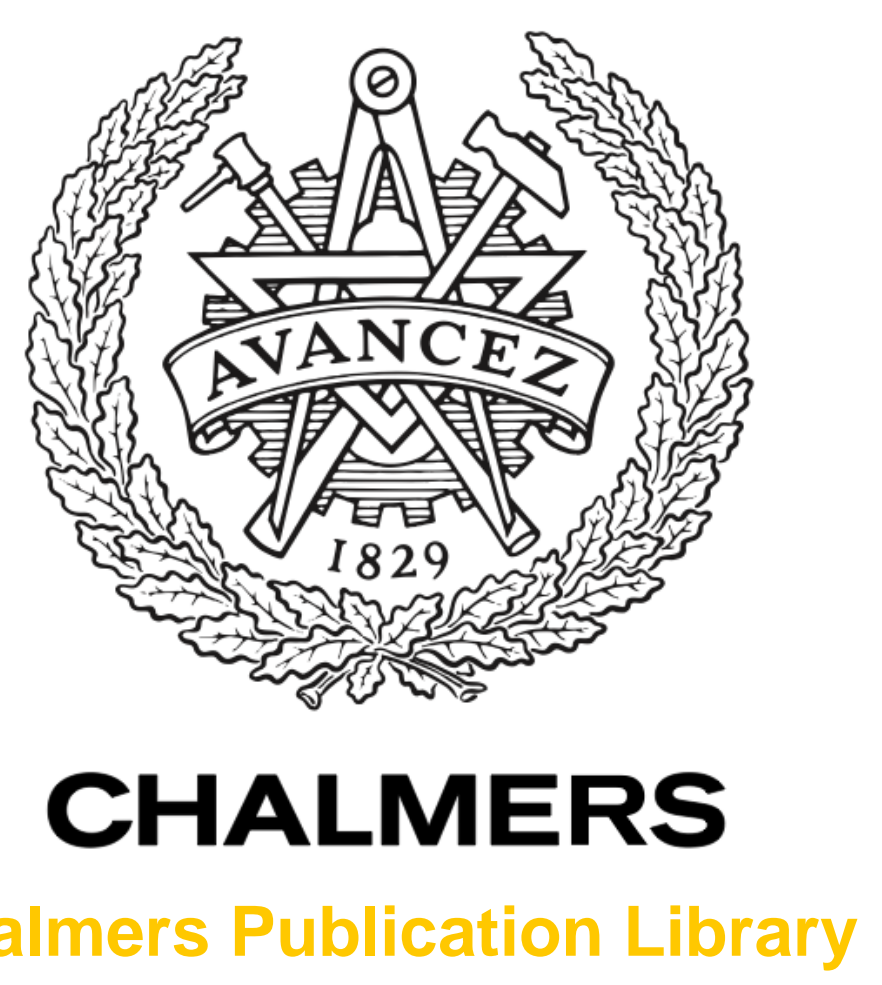

Chalmers Publication Library

\title{
Variational Inference-based Positioning with Nondeterministic Measurement Accuracies and Reference Location Errors
}

This document has been downloaded from Chalmers Publication Library $(\mathrm{CPL})$. It is the author's version of a work that was accepted for publication in:

IEEE Transactions on Mobile Computing (ISSN: 1536-1233)

Citation for the published paper:

Zhou, B. ; Chen, Q. ; Wymeersch, H. et al. (2016) "Variational Inference-based Positioning with Nondeterministic Measurement Accuracies and Reference Location Errors". IEEE

Transactions on Mobile Computing

http://dx.doi.org/10.1109/TMC.2016.2640294

Downloaded from: http://publications.lib.chalmers.se/publication/248926

Notice: Changes introduced as a result of publishing processes such as copy-editing and formatting may not be reflected in this document. For a definitive version of this work, please refer to the published source. Please note that access to the published version might require a subscription. 


\title{
Variational Inference-based Positioning with Nondeterministic Measurement Accuracies and Reference Location Errors.
}

\author{
Bingpeng Zhou, Qingchun Chen, Henk Wymeersch, Pei Xiao and Lian Zhao
}

\begin{abstract}
Cooperative network localization plays an important role in wireless sensor network (WSN), wherein neighboring sensor nodes will help each other to calibrate their locations. However, due to the dynamic wireless propagation environment and different surroundings, the measurement accuracy at different network nodes is different and varies over time. In this paper, the uncertainties in both measurement accuracy and reference node locations are considered to account for the impact of different surrounding environments and the initial node location errors on the cooperative network localization. A mean-field variational inference-based positioning (VIP) algorithm is proposed for cooperative network localization. The mechanism of the proposed VIP algorithm, the convergence properties, implementation complexity, and the parallel implementation structure are presented to show that the VIP algorithm provides an effective mechanism to incorporate and share the localization information among all network nodes for an improved localization performance. Finally, a concise Cramer-Rao lower bound (CRLB) is derived to reveal the principle of localization error propagation. It is disclosed that the localization error propagation principle is similar to the Ohm's Law in circuit theory, which provides a new insight into the impact of the measurement accuracy, the reference node location errors and the number of reference nodes on the cooperative network localization performance.
\end{abstract}

Index Terms-Nondeterministic Measurement Accuracy, Reference Node Location Error, Cooperative Network Localization, CRLB.

\section{INTRODUCTION}

W IRELESS sensor network (WSN)-based cooperative localization is an effective solution to enable the location-aware services, such as location-aware security [1], [2], warehousing management, environmental monitoring and shopping mall navigation, which revolutionize the way people search, locate and navigate the points of interest inside buildings [3], [4].

Since the global positioning system (GPS) signal is usually unavailable in indoor environments [5], [6], it is of practical importance to explore the dedicated WSN-based cooperative localization, where various measurement signals can be used, such as received signal strength (RSS) [7], time of arrival (TOA) [8], [9], [10], [11] and angle of arrival (AOA) [12]. However, there are several technical challenges still remaining for the wireless network localization. First, the localization cooperation with neighboring nodes is required due to the limited number of anchor nodes in the network. The reference node locations are inaccurate, owing to errors in their initial location acquisitions. Hence, the reference node location uncertainties are critical issues to be considered in practical cooperative localization. Second, the measurement accuracies associated with different reference nodes are

- Bingpeng Zhou and Qingchun Chen are with the School of Information Science and Technology, Southwest Jiaotong University, Chengdu, P.R. China,610031. E-mail: zhoubingpeng@163.com, qcchen@swjtu.edu.cn.

- Henk Wymeersch is with Chalmers University of Technology, Gothenberg, Sweden.henkw@chalmers.se.

- Pei Xiao is with University of Surrey, Guildford, Surrey GU2 7XH, UK. E-mail: p.xiao@surrey.ac.uk

- Lian Zhao is with Ryerson University, Toronto, Ontario, Canada, M5B 2K3. E-mail: 15zhao@ryerson.ca

Manuscript received 21 March, 2015; accepted 12 December, 2016. different, due to their different surrounding environments, device orientations, strengths of thermal noises and levels of shadow fading. ${ }^{1}$ Moreover, in a dynamic environment (such as the shopping mall crowded with moving people), the measurement accuracy varies over time, and its real value is thus difficult to obtain. Hence, the measurement accuracies of different reference nodes are no longer identical, deterministic and known. In this case, a hyper-prior model is needed to characterize the randomness of nondeterministic measurement accuracy. Consequently, in order to achieve optimal performance, both reference node location error and measurement accuracy randomness should be considered in cooperative localization.

A multitude of localization schemes have been proposed with different measurement data (e.g., node connectivity [6], multihop number [15], [16], AOA [12], TOA [8], [9], [10], [11], time difference of arrival [17], frequency difference of arrival [18], gain ratios of arrival and range differences of arrival [19]), under various constraints $(e . g$. , the reference node location errors [20], [21] and radio wave measurement [6]) in different scenarios (e.g., underwater [22], indoor [23], outdoor [24], dynamic tracking [25] and acoustic environment [26]). For example, the reference node location error was studied in [20] and [21], where the importance sampling and expectation-maximization-based positioning methods were proposed respectively to deal with the anchor node location errors. The corresponding Cramer-Rao lower bound (CRLB) was derived in [20] to quantify the impact

\footnotetext{
${ }^{1}$ Here, the measurement accuracy is defined as the statistical distribution precision of additive measurement errors, i.e., the inverse of the measurement error covariance [13], [14], based on the statistical theory.
} 
of reference node location errors. The CRLB associated with gain ratios of arrival in conjunction with range differences of arrival was analyzed in [19] to study the effect of reference node location errors. A multihop localization scheme based on the evidence theory was proposed in [15] to deal with the errors in the reference node locations. The effect of reference node location errors was also analysed in [18], wherein the time and frequency difference of arrival measurements were utilized to perform the source localization. In [27], the performance limits of the RSS-based and the node connectivity-based localization were investigated to clarify which strategy should be employed, and to find out the optimal threshold for confirming the connectivity of two network nodes. There are other methods, such as the variational message passing [28], optimization methods [29], belief propagation [30], which can be employed to enable accurate localization in the presence of the reference node location errors. However, almost all of them assume the statistical precision parameters of measurement signal errors at different nodes are identical, deterministic and known. ${ }^{2}$ Harnessing the randomness of statistical measurement accuracy is crucial not only for the analysis of localization performance, but also for the development of efficient localization algorithm.

In this paper, we assume the measurement accuracy of different node pairs is nondeterministic. We use a Wishart density, which is the conjugate priori of the precision of a Gaussian variable, to characterize the nondeterministic measurement accuracy. The Gaussian density is employed to model the errors in the initial node locations. On this basis, a mean-field variational inference-based positioning (VIP) scheme is developed to share the measurement data and prior location information among all network nodes for localization performance enhancement. Moreover, a concise CRLB is derived to reveal the principle of localization error propagation. The impact of reference node location errors and the measurement accuracy on the localization performance is also analyzed. It is disclosed that, the error propagation mechanism resembles the Ohm's Law in circuit theory, which provides a new insight into the impacts of the measurement accuracy, the reference node location errors and the number of reference nodes on the localization performance. The proposed VIP algorithm is applied to the RSS-based network localization, due to its model simplicity and popular accessibility to WSNs.

The contributions of this paper are two-fold.

- A mean-field VIP scheme is proposed for cooperative network localization, accounting for both the measurement accuracy randomness and the reference node location errors. The VIP scheme can reap further performance benefits from the global localization cooperation (i.e., sharing of measurement data and priori location information among all network nodes) and the variational messages propagation within the associated Markov Blanket variables.

- A concise CRLB is derived to reveal the localization error propagation mechanism. In addition, the impact of the number of reference nodes, the reference

\footnotetext{
${ }^{2}$ We use "measurement accuracy" to stand for "statistical precision parameters of measurement signal error" thereafter for brevity purpose, whenever there is no confusion.
}

node location errors and the measurement accuracies are asymptotically analyzed to show how these dependent factors dominate the cooperative localization performance. It indicates the performance limits for a localization algorithm. Moreover, the theoretical analysis results can guide the algorithm design to strike a balance between localization performance and implementation overhead.

The remainder of this paper is organized as follows. Section 2 describes the system model and the problem concerned. A mean-field VIP algorithm is proposed in Section 3. The performance limits of VIP are analysed in Section 4. Simulation and experiment results are presented in Sections 5 and 6, respectively. Finally, Section 7 concludes the paper.

\section{System Model}

\subsection{WSN Model}

We consider a static WSN, as shown in Fig. 1, where all sensor nodes are assumed to be uniformly distributed inside the deployment area and the total number of sensor nodes is assumed to be $M$. In addition, there are only inaccurate nodes (no anchors) inside the whole area, and all sensor nodes are supposed to be localized with the cooperation of other nodes. We assume that, all sensor node locations are inaccurate due to errors in acquisition of their initial locations. The true (but unknown) location of the $i$ th sensor node is denoted by a $\mathfrak{D}$-dimensional column vector $\mathbf{s}_{i}$, while the coarse location (inaccurate location with a precision $\mathbf{U}_{i}$ ) recorded by the $i$ th sensor node is denoted by $\boldsymbol{\mu}_{i}$. The coarse location $\boldsymbol{\mu}_{i}$ and precision $\mathbf{U}_{i}$ are recorded by the sensor node and will be reported to the objective node (the node to be located). In general, the true location $\mathbf{s}_{i}$ is modeled as a Gaussian distribution with the center $\boldsymbol{\mu}_{i}$ and the precision $\mathbf{U}_{i}$, namely, ${ }^{3}[31]$

$$
\mathbf{s}_{i} \sim \mathcal{N}\left(\mathbf{s}_{i} \mid \boldsymbol{\mu}_{i}, \mathbf{U}_{i}\right), \forall i=1: M,
$$

where we assume sensor location precision $\mathbf{U}_{i}$ is independent with each other. The location uncertainty is defined as the inverse of the location precision matrix $\mathbf{U}_{i}$.

Considering the localization of sensor node $\mathbf{s}_{i}$ (the objective node), we assume $\mathbf{s}_{i}$ is within the sensing range $r_{s}$ of $M_{i}$ nearby nodes (reference nodes), and we define the index set of these reference nodes as

$$
\Psi_{i} \doteq\left\{j:\left\|\mathbf{s}_{j}-\mathbf{s}_{i}\right\|_{2}<r_{s}, \forall j \neq i\right\},
$$

where the symbol $\|\bullet\|_{2}$ denotes the $\ell_{2}$-norm on a vector. Hence, we have the set cardinal number $\left|\Psi_{i}\right|=M_{i}$.

\subsection{Measurement Model}

The general measurement model of cooperative wireless localization can be given by

$$
\mathrm{z}_{i, j}=h\left(\mathbf{s}_{i}, \mathbf{s}_{j}\right)+\epsilon_{i, j}, \forall j \in \Psi_{i} \text { and } \forall i=1: M,
$$

where $\mathrm{z}_{i, j}$ denotes the associated measurement from $\mathbf{s}_{j}$ to $\mathbf{s}_{i}$, and $\epsilon_{i, j}$ denotes the additive measurement noise.

${ }^{3}$ Although we assume no anchor in the deployment area, the anchor node location can also be characterized by Eq. (1) when the location precision $\mathbf{U}_{i}$ approaches infinity. Hence, this location model subsumes the case with anchor node(s). 


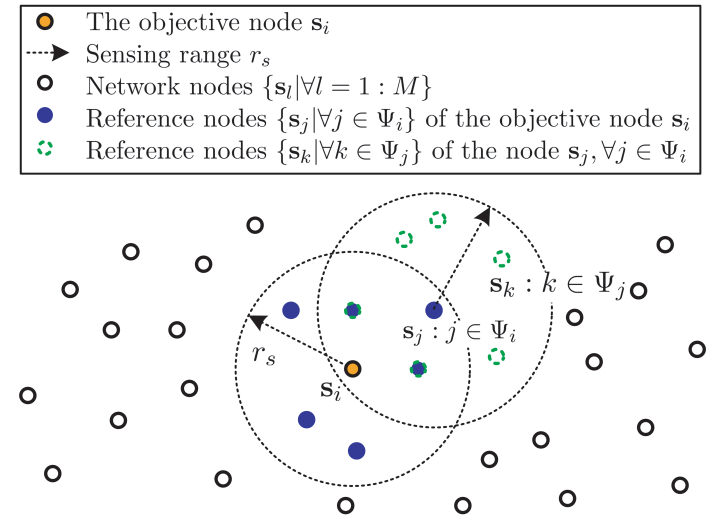

Fig. 1. Illustration of the WSN deployment. Those nodes inside sensing range $r_{s}$ around $\mathbf{s}_{i}$, form the reference node cluster $\left\{\mathbf{s}_{j} \mid \forall j \in \Psi_{i}\right\}$

In particular, $h\left(\mathbf{s}_{i}, \mathbf{s}_{j}\right)$ is defined as the measurement function, which depends on the distance $\left\|\mathbf{s}_{i}-\mathbf{s}_{j}\right\|_{2}$ (rangebased methods) [32], the angle $\angle\left(\mathbf{s}_{i}, \mathbf{s}_{j}\right)$ (direction-based methods) [33] or the connectivity c $\left(\mathbf{s}_{i}, \mathbf{s}_{j}\right)$ [27] of two nodes.

In this paper, the following analysis is valid for the TOA, AOA and particularly the RSS-based localization [11], [12], [7], [25], where the associated measurement function $h\left(\mathbf{s}_{i}, \mathbf{s}_{j}\right)$ can be specified, respectively, as [32], ${ }^{4,5}$

$$
\begin{aligned}
h_{\mathrm{TOA}}\left(\mathbf{s}_{i}, \mathbf{s}_{j}\right) & =\left\|\mathbf{s}_{i}-\mathbf{s}_{j}\right\|_{2}, \\
h_{\mathrm{RSS}}\left(\mathbf{s}_{i}, \mathbf{s}_{j}\right) & =\phi-10 \gamma \log _{10}\left\|\mathbf{s}_{i}-\mathbf{s}_{j}\right\|_{2}, \\
h_{\mathrm{AOA}}\left(\mathbf{s}_{i}, \mathbf{s}_{j}\right) & =\varphi_{j}+\frac{180}{\pi} \operatorname{actan}\left(\frac{\left[\mathbf{s}_{i}-\mathbf{s}_{j}\right]_{2}}{\left[\mathbf{s}_{i}-\mathbf{s}_{j}\right]_{1}}\right),
\end{aligned}
$$

wherein $\phi=P_{T}-L_{0}+10 \gamma \log _{10} d_{0}$ and $P_{T}$ is the transmit power, $L_{0}$ denotes the path loss associated with the reference distance $d_{0}, \gamma$ denotes the path loss exponent [36]. In addition, $[\mathbf{x}]_{k}$ stands for the $k$ th $(k=1,2)$ element of a two dimensional vector $\mathbf{x}$, and $\varphi_{j}$ stands for the direction of the antenna main lobe. Unless otherwise stated, we use $h\left(\mathbf{s}_{i}, \mathbf{s}_{j}\right)$ to denote the general range-based measurement function.

The measurement error $\epsilon_{i, j}$ is generally modeled as a zero-mean Gaussian noise, i.e., $\epsilon_{i, j} \sim \mathcal{N}\left(\epsilon_{i, j} \mid 0, \mathrm{w}_{i, j}\right)$, where $\mathrm{w}_{i, j}$ is defined as the measurement precision (or accuracy). In practice, the precision of different measurement noise is different due to different levels of shadowing, different device orientation and different obstructions [37]. In addition, due to the dynamic surrounding environment (such as the shopping mall crowded with moving people), the measurement precision $\mathrm{w}_{i, j}$ changes over time and it is difficult to obtain its exact value. Hence, we assume the measurement precision $\mathrm{w}_{i, j}$ associated with different node pairs $\left(\mathbf{s}_{i}, \mathbf{s}_{j}\right)$ is nondeterministic and unknown.

Hence, a hyperpriori model is needed to characterize the randomness of nondeterministic measurement precision $\mathrm{w}_{i, j}$. In Bayesian statistics, the Wishart density is the conjugate priori of the precision of a Gaussian distribution, thus it can be used to characterize the hyperpriori of the precision

\footnotetext{
${ }^{4}$ For the TOA-based localization, we have considered the case that the non-light-of-sight signal can be identified and removed by the identification methods [6], [34] and its positive ranging error can also be mitigated [35]. The network timer is also assumed to be synchronized.

${ }^{5}$ For the AOA-based localization (see Eq. (6)), we assume the scenario is in a 2-dimensional Euclid space, i.e., $\mathfrak{D}=2$.
}

parameter of a Gaussian variable [38], [39]. It can not only facilitate the associated theoretical analysis, but also guarantee a reasonable assumption that the priori distribution and the posteriori distribution of measurement precision fall in the same distribution family [40]. The Wishart density is also commonly assumed for the precision of a Gaussian variable, for instance in [41], [42]. In this paper, we also apply the Wishart density to model $\mathrm{w}_{i, j}$, i.e.,

$$
\mathrm{w}_{i, j} \sim \mathcal{W}\left(\mathrm{w}_{i, j} \mid \mathrm{W}, \psi\right), \forall j \in \Psi_{i} \text { and } \forall i=1: M,
$$

where the positive scalar $\mathrm{W}$ denotes its scale and $\psi$ denotes its degree of freedom (DoF). This model is validated by real experiments presented in Section 5 (see Fig. 10(a)). Note that, this model subsumes the case where the measurement precision $\mathrm{w}_{i, j}$ is deterministic and known, when $\psi \mathrm{W}^{2} \rightarrow 0$.

\subsection{Problem Formulation}

Given each network node's coarse location $\mu_{i}$, precision $\mathbf{U}_{i}$ and its measurement $\mathrm{z}_{i, j}, \forall j \in \Psi_{i}, \forall i=1: M$, the task is to localize all network node positions and reveal the localization performance limits, using limited state information of measurement precision $\mathrm{w}_{i, j}$.

\section{Algorithm Design}

In this section, by exploiting the underlying statistics of both sensor node locations and measurement precisions, a meanfield VIP algorithm is developed.

\subsection{Statistical Model}

Prior to presenting algorithm details, the objective function and some variable definitions are introduced at first.

Let $\boldsymbol{c}_{i}=\operatorname{vec}\left[\mathbf{s}_{j}\right]_{\forall j \in \Psi_{i}}$ be the cluster of reference nodes, where $\operatorname{vec}\left[\bullet_{j}\right]_{\forall j \in \Psi_{i}}$ yields a column vector stacked by all components $\left\{\bullet_{j}: \forall j \in \Psi_{i}\right\}$. The measurements of node $\mathbf{s}_{i}$ from $\Psi_{i}$ are stacked as $\mathbf{z}_{i}=\operatorname{vec}\left[\mathrm{z}_{i, j}\right]_{\forall j \in \Psi_{i}}$. We define a measurement precision vector as $\boldsymbol{\omega}_{i}=\operatorname{vec}\left[\mathrm{w}_{i, j}\right]_{\forall j \in \Psi_{i}}$. Considering the localization of senor node $\mathbf{s}_{i}$ (also called the objective node), we define the associated complement variable as $\boldsymbol{\alpha}_{i}=\left[\mathbf{s}_{i}^{\top}, \boldsymbol{c}_{i}^{\top}, \boldsymbol{\omega}_{i}\right]^{\top}$ with $\left(M_{i} \mathfrak{D}+\mathfrak{D}+M_{i}\right)$ dimensions, which incorporates all nondeterministic variables associated with localization of $\mathbf{s}_{i}$. Meanwhile, let $\boldsymbol{\alpha}_{i}^{n}$ denote the $n$th individual variable in the complete variable $\left\{\boldsymbol{\alpha}_{i}\right\}$.

Consequently, assuming the measurements conditioned on $\boldsymbol{\alpha}_{i}$ are mutually independent, the likelihood distribution associated with $\boldsymbol{\alpha}_{i}$ is specified as

$$
p\left(\mathbf{z}_{i} \mid \boldsymbol{\alpha}_{i}\right)=\prod_{j \in \Psi_{i}} \frac{\left|\mathrm{w}_{i, j}\right|^{\frac{1}{2}}}{\sqrt{2 \pi}} \exp \left(-\frac{1}{2} \mathrm{w}_{i, j}\left(\mathrm{z}_{i, j}-h\left(\mathbf{s}_{i}, \mathbf{s}_{j}\right)\right)^{2}\right) .
$$

Hence, the posteriori distribution of $\boldsymbol{\alpha}_{i}$ is cast as

$$
\begin{aligned}
& p\left(\boldsymbol{\alpha}_{i} \mid \mathbf{z}_{i}\right) \propto p\left(\mathbf{z}_{i} \mid \boldsymbol{\alpha}_{i}\right) p\left(\boldsymbol{\alpha}_{i}\right) \\
= & \prod_{j \in \Psi_{i}} \frac{\left|\mathrm{w}_{i, j}\right|^{\frac{1}{2}}}{\sqrt{2 \pi}} \exp \left(-\frac{1}{2} \mathrm{w}_{i, j}\left(\mathrm{z}_{i, j}-h\left(\mathbf{s}_{i}, \mathbf{s}_{j}\right)\right)^{2}\right) \\
& \cdot \mathcal{N}\left(\mathbf{s}_{j} \mid \boldsymbol{\mu}_{j}, \mathbf{U}_{j}\right) \mathcal{W}\left(\mathrm{w}_{i, j} \mid \mathrm{W}, \psi\right) \cdot \mathcal{N}\left(\mathbf{s}_{i} \mid \boldsymbol{\mu}_{i}, \mathbf{U}_{i}\right),
\end{aligned}
$$

where $\propto$ denotes the left is proportional to the right. 
In particular, the posteriori distribution associated with senor node location $\mathbf{s}_{i}$ is marginalized as

$$
\begin{aligned}
& p\left(\mathbf{s}_{i} \mid \mathbf{z}_{i}\right) \propto p\left(\mathbf{s}_{i}\right) \int p\left(\mathbf{z}_{i} \mid \boldsymbol{\alpha}_{i}\right) p\left(\widetilde{\mathbf{s}}_{i}\right) \mathrm{d} \widetilde{\mathbf{s}}_{i} \\
&= \mathcal{N}\left(\mathbf{s}_{i} \mid \boldsymbol{\mu}_{i}, \mathbf{U}_{i}\right) \\
& \cdot \prod_{j \in \Psi_{i}} \iint \frac{\left|\mathrm{w}_{i, j}\right|^{\frac{1}{2}}}{\sqrt{2 \pi}} \exp \left(-\frac{1}{2} \mathrm{w}_{i, j}\left(\mathrm{z}_{i, j}-h\left(\mathbf{s}_{i}, \mathbf{s}_{j}\right)\right)^{2}\right) \\
& \cdot \mathcal{N}\left(\mathbf{s}_{j} \mid \boldsymbol{\mu}_{j}, \mathbf{U}_{j}\right) \mathcal{W}\left(\mathrm{w}_{i, j} \mid \mathrm{W}, \psi\right) \mathrm{d} \mathbf{s}_{j} \mathrm{dw}_{i, j},
\end{aligned}
$$

where $\widetilde{\mathbf{s}}_{i}$ is defined as the complementary variable set of $\mathbf{s}_{i}$, i.e., $\widetilde{\mathbf{s}}_{i}=\boldsymbol{\alpha}_{i} \backslash \mathbf{s}_{i}$, by introducing set partition $\left\{\boldsymbol{\alpha}_{i}\right\}=\left\{\mathbf{s}_{i}, \widetilde{\mathbf{s}}_{i}\right\}$.

The localization of node $\mathbf{s}_{i}$ in terms of either minimum mean squared error or maximum posteriori density is based on the above marginalized posteriori $p\left(\mathbf{s}_{i} \mid \mathbf{z}_{i}\right)$. However, due to the nonlinear measurement function $h\left(\mathbf{s}_{i}, \mathbf{s}_{j}\right)$ and nondeterministic measurement precision $\mathrm{w}_{i, j}$, there is no closedform expression of $p\left(\mathbf{s}_{i} \mid \mathbf{z}_{i}\right)$, (i.e., it can not be calculated out with limited amount of common operations and functions [43]), which complicates the localization realization. Hence, we resort to the variational Bayesian inference (VBI) method [44] to solve this problem.

\subsection{Theoretical Basis}

Considering the intractable posteriori $p\left(\mathbf{s}_{i} \mid \mathbf{z}_{i}\right), \forall i=1: M$, we use a set of another distributions $\left\{q\left(\boldsymbol{\alpha}_{i}^{(n)}\right) \mid \forall \boldsymbol{\alpha}_{i}^{(n)} \in \boldsymbol{\alpha}_{i}\right\}$ to approximate the complete variable-associated posteriori $p\left(\boldsymbol{\alpha}_{i}^{(n)} \mid \mathbf{z}_{i}\right)$, such that the Kullback-Leibler divergence (KLD) between the joint density $q\left(\boldsymbol{\alpha}_{i}\right)$ and the objective posteriori density $p\left(\boldsymbol{\alpha}_{i} \mid \mathbf{z}_{i}\right)$ is minimized. ${ }^{6}$ The utilized KLD metric is defined as [44]

$$
D_{\mathrm{KL}}[q \| p]=\int q\left(\boldsymbol{\alpha}_{i}\right) \ln \frac{q\left(\boldsymbol{\alpha}_{i}\right)}{p\left(\boldsymbol{\alpha}_{i} \mid \mathbf{z}_{i}\right)} \mathrm{d} \boldsymbol{\alpha}_{i},
$$

where $q\left(\boldsymbol{\alpha}_{i}\right)$ is assumed to be factorized as [44]

$$
q\left(\boldsymbol{\alpha}_{i}\right)=q\left(\mathbf{s}_{i}\right) \prod_{j \in \Psi_{i}} q\left(\mathbf{s}_{j}\right) q\left(\mathrm{w}_{i, j}\right),
$$

where $q\left(\mathbf{s}_{i}\right)$ stands for the approximation to the individual posteriori $p\left(\mathbf{s}_{i} \mid \mathbf{z}_{i}\right)$, and so are $q\left(\mathbf{s}_{j}\right)$ and $q\left(\mathrm{w}_{i, j}\right)$.

Based on the mean-field VBI theory, the optimal variational approximation $q\left(\boldsymbol{\alpha}_{i}^{n}\right)$ of each individual variable $\boldsymbol{\alpha}_{i}^{n}$, $\forall \boldsymbol{\alpha}_{i}^{n} \in\left\{\boldsymbol{\alpha}_{i}\right\}$, is finally derived as

$$
q\left(\boldsymbol{\alpha}_{i}^{n}\right) \propto \exp \left(\left\langle\ln p\left(\boldsymbol{\alpha}_{i}^{n}, \mathcal{B}\left(\boldsymbol{\alpha}_{i}^{n}\right)\right)\right\rangle_{\mathcal{B}\left(\boldsymbol{\alpha}_{i}^{n}\right)}\right),
$$

where $\left\langle g\left(\boldsymbol{\alpha}_{i}^{n}\right)\right\rangle_{\boldsymbol{\alpha}_{i}^{n}}=\int g\left(\boldsymbol{\alpha}_{i}^{n}\right) q\left(\boldsymbol{\alpha}_{i}^{n}\right) \mathrm{d} \boldsymbol{\alpha}_{i}^{n}$, and $\mathcal{B}\left(\boldsymbol{\alpha}_{i}^{n}\right)$ stands for the Markov blanket (MB) [48] associated with variable $\boldsymbol{\alpha}_{i}^{n}, \forall \boldsymbol{\alpha}_{i}^{n} \in\left\{\boldsymbol{\alpha}_{i}\right\}$, which can be specified as

$$
\begin{aligned}
\mathcal{B}\left(\mathbf{s}_{i}\right) & =\left\{\mathbf{s}_{j}, \mathrm{w}_{i, j}, \mathrm{z}_{i, j}: \forall j \in \Psi_{i}\right\}, \forall i=1: M, \\
\mathcal{B}\left(\mathrm{w}_{i, j}\right) & =\left\{\mathbf{s}_{i}, \mathbf{s}_{j}, \mathrm{z}_{i, j}\right\}, \forall j \in \Psi_{i}, \text { and } \forall i=1: M .
\end{aligned}
$$

Then, based on Eq. (12), each variational approximation $q\left(\mathbf{s}_{i}\right)$ and $q\left(\mathrm{w}_{i, j}\right)$ to the corresponding posteriori $p\left(\mathbf{s}_{i} \mid \mathbf{z}_{i}\right)$ and $p\left(\mathrm{w}_{i, j} \mid \mathbf{z}_{i}\right)$, respectively, can be iteratively calculated in parallel and gradually converge, as addressed below.

${ }^{6}$ There are a multitude of other methods to find the approximation of a complicated posteriori density function, such as Monte Carlo sampling [20], [45], unscented transformation [46], Laplace approximation [47] and linearization [45].

\subsection{Algorithm Formulation}

At each VBI iteration of the VIP algorithm, when deriving $q\left(\boldsymbol{\alpha}_{i}^{n}\right)$ we assume other approximations $q\left(\boldsymbol{\alpha}_{i}^{m}\right), \forall m \neq n$, are determined. Based on the mean-field VBI method, the variational approximations in VIP are updated as follows.

(i) Update of $q\left(\mathbf{s}_{i}\right)$ about Sensor Node Location: We first consider the update of node $\mathbf{s}_{i}, \forall i=1: M$. According to Eq. (12), given $q\left(\mathbf{s}_{j}\right)$ and $q\left(\mathrm{w}_{i, j}\right), \forall j \in \Psi_{i}$, the variational distribution $q\left(\mathbf{s}_{i}\right)$ is approximated by a variational particle set $\left\{\mathbf{s}_{i}^{(\tau)}, \wp_{i}^{(\tau)} \mid \forall \tau=1: N_{s}\right\}$ as follows (please see the detailed derivation in APPENDIX A),

$$
q\left(\mathbf{s}_{i}\right) \approx \sum_{\tau=1: N_{s}} \wp_{i}^{(\tau)} \delta\left(\mathbf{s}_{i}-\mathbf{s}_{i}^{(\tau)}\right),
$$

where $N_{s}$ denotes the number of particles, and $\delta$ denotes the Dirac function. In addition, $\mathbf{s}_{i}^{(\tau)}$ stands for the supporting point of particle, which is drawn from its priori $p\left(\mathbf{s}_{i}\right)$, i.e.,

$$
\mathbf{s}_{i}^{(\tau)} \sim p\left(\mathbf{s}_{i}\right),
$$

and $\wp_{i}^{(\tau)}$ denotes its weight, which is given by

$$
\wp_{i}^{(\tau)} \propto \prod_{\substack{j \in \Psi_{i}, k=1: N_{s}}}^{\exp \left(-\frac{1}{2} \wp_{j}^{(k)}\left\langle\mathrm{w}_{i, j}\right\rangle\left(\mathrm{z}_{i, j}-h\left(\mathbf{s}_{i}^{(\tau)}, \mathbf{s}_{j}^{(k)}\right)\right)^{2}\right)} \underbrace{}_{\mathcal{P}_{i}\left(\mathbf{s}_{j}^{(k)}\right)}
$$

where the approximation expectation $\left\langle\mathrm{w}_{i, j}\right\rangle=\psi_{i, j}^{\sharp} \mathrm{W}_{i, j}^{\sharp}$, while $W_{i, j}^{\sharp}$ and $\psi_{i, j}^{\sharp}$ denote the posterior scale and the posterior DoF of a Wishart distribution, respectively, i.e., $q\left(\mathrm{w}_{i, j}\right)=\mathcal{W}\left(\mathrm{w}_{i, j} \mid \mathrm{W}_{i, j}^{\sharp}, \psi_{i, j}^{\sharp}\right)$ (see Eq. (20)). A simple notation $\left\langle\mathrm{w}_{i, j}\right\rangle$ is used in (17) to stand for $\left\langle\mathrm{w}_{i, j}\right\rangle_{\mathrm{w}_{i, j}}$, when the two variables involved in the operator $\langle\bullet\rangle$. are the same. Please see APPENDIX A for the detailed derivation.

We can find out that, each particle $\mathbf{s}_{j}^{(k)}$ of reference node $\mathbf{s}_{j}$ carries variational message $\mathcal{P}_{i}\left(\mathbf{s}_{j}^{(k)}\right)$. All these variational messages $\left\{\mathcal{P}_{i}\left(\mathbf{s}_{j}^{(k)}\right)\right\}_{\substack{\forall k=1: N_{s} \\ \forall j \in \Psi_{i}}}$ carried by different particles of different reference nodes jointly enhance the location confidence $q\left(\mathbf{s}_{i}\right)$ of objective node $\mathbf{s}_{i}$. In addition, at each VBI iteration associated with each node, all particle weights $\left\{\wp_{i}^{(\tau)} \mid \forall \tau=1: N_{s}\right\}$ can be updated in parallel (see more details in Section 3.5.(ii)).

Given the variational particles derived as above, the sensor location and the associated location precision can be determined, according to an asymptotic minimum mean squared error criterion, as below

$$
\begin{aligned}
\widehat{\mathbf{s}}_{i} & =\sum_{n=1: N_{s}} \wp_{i}^{(n)} \mathbf{s}_{i}^{(n)}, \\
\widehat{\mathbf{U}}_{i} & =\left(\sum_{n=1: N_{s}} \wp_{i}^{(n)}\left(\mathbf{s}_{i}^{(n)}-\widehat{\mathbf{s}}_{i}\right)\left(\mathbf{s}_{i}^{(n)}-\widehat{\mathbf{s}}_{i}\right)^{\top}\right)^{-1} .
\end{aligned}
$$

Namely, in addition to the node location estimation $\widehat{\mathbf{s}}_{i}$, the location precision $\widehat{\mathbf{U}}_{i}$ can also be iteratively updated.

As for the approximation update $q\left(\mathbf{s}_{j}\right)$ associated with the reference node $\mathbf{s}_{j}, \forall j \in \Psi_{i}$, it is equivalent to the update of $q\left(\mathbf{s}_{i}\right)$ in (15), where the approximate distribution $q\left(\mathbf{s}_{j}\right)$ is derived by integrating the posterior information (e.g., $q\left(\mathbf{s}_{i}\right)$ and $\left.q\left(\mathrm{w}_{i, j}\right)\right)$ propagated from its $\mathrm{MB} \mathcal{B}\left(\mathbf{s}_{j}\right)$. 
In fact, the VIP algorithm combines both the variational inference and importance sampling to deduce the complicated posteriori distribution $p\left(\mathbf{s}_{i} \mid \mathbf{z}_{i}\right)$, and to enable a feasible network localization scheme, which is different from the traditional particle-assisted approximation method, e.g., the localization scheme in [20]. The variational iterations can gradually improve the particle efficiency with low approximation error (see Section 3.5.(i) for more details).

(ii) Update of $q\left(\mathrm{w}_{i, j}\right)$ about Measurement Precision: We now formulate the update of approximate posteriori $q\left(\mathrm{w}_{i, j}\right)$. Assuming $q\left(\mathbf{s}_{i}\right)$ and $q\left(\mathbf{s}_{j}\right), \forall i=1: M$ and $\forall j \in \Psi_{i}$, have been determined in the previous iteration, the approximate distribution $q\left(\mathrm{w}_{i, j}\right)$ can be derived as the following Wishart density (see APPENDIX B for the details),

$$
\begin{aligned}
q\left(\mathrm{w}_{i, j}\right) & \propto\left|\mathrm{w}_{i, j}\right|^{\frac{(\psi+1)-2}{2}} \exp \left(-\frac{1}{2}\left(\mathrm{~W}_{i, j}^{\sharp}\right)^{-1} \mathrm{w}_{i, j}\right) \\
& \stackrel{\mathrm{d}}{=} \mathcal{W}\left(\mathrm{w}_{i, j} \mid \mathrm{W}_{i, j}^{\sharp}, \psi_{i, j}^{\sharp}\right),
\end{aligned}
$$

where $\stackrel{\mathrm{d}}{=}$ denotes the equality in the sense of distribution. In addition, the posteriori $\operatorname{DoF} \psi_{i, j}^{\sharp}=\psi+1$, and we have

$$
\begin{aligned}
& \mathrm{W}_{i, j}^{\sharp}=\left(\left\langle\left(\mathrm{z}_{i, j}-h\left(\mathbf{s}_{i}, \mathbf{s}_{j}\right)\right)^{2}\right\rangle_{\mathbf{s}_{i}, \mathbf{s}_{j}}+\mathrm{W}^{-1}\right)^{-1}, \\
\approx & \left(\sum_{\substack{k=1: N_{s} \\
\tau=1: N_{s}}} \wp_{i}^{(\tau)} \wp_{j}^{(k)}\left(\mathrm{z}_{i, j}-h\left(\mathbf{s}_{i}^{(\tau)}, \mathbf{s}_{j}^{(k)}\right)\right)^{2}+\mathrm{W}^{-1}\right)^{-1},
\end{aligned}
$$

where the variational particles $\left\{\mathbf{s}_{i}^{(\tau)}, \wp_{i}^{(\tau)} \mid \forall \tau=1: N_{s}\right\} \sim$ $q\left(\mathbf{s}_{i}\right)$ and $\left\{\mathbf{s}_{j}^{(k)}, \wp_{j}^{(k)} \mid \forall k=1: N_{s}\right\} \sim q\left(\mathbf{s}_{j}\right)$ are employed to approximate the complicated term in Eq. (21).

Hence, the nondeterministic measurement precision can be updated as its posteriori expectation in the following way

$$
\left\langle\mathrm{w}_{i, j}\right\rangle=\psi_{i, j}^{\sharp} \mathrm{W}_{i, j}^{\sharp},
$$

which is also referred to as its asymptotic minimum mean square error estimation, $i . e ., \widehat{\mathrm{w}}_{i, j}=\left\langle\mathrm{w}_{i, j}\right\rangle$.

\subsection{Algorithm Realization}

The VIP scheme can be performed not only in a centralized computation center (i.e., a centralized localization manner) but also by each individual sensor node (i.e., a distributed localization manner). For a centralized localization strategy, once all the measurement data and priori information of network node locations are gathered, the computation center can act on the global data (measurements, variational message, node location information) to localize network nodes in parallel (see Section 3.5.(ii)). On the other hand, for a distributed localization strategy, each sensor node $\mathbf{s}_{i}$ can derive its own location through exchanging the variational message $\left\{\mathcal{P}_{i}\left(\mathbf{s}_{j}^{(k)}\right) \mid \forall k=1: N_{s}\right\}$ with its nearby nodes $\left\{\mathbf{s}_{j} \mid \forall j \in \Psi_{i}\right\}$ only. Of course, in such a strategy, each node should be provisioned with sufficient hardware to afford the VIP calculation. The algorithm complexity will be discussed in Section 3.5.(iv).

Once obtaining $\left\{\mathcal{P}_{i}\left(\mathbf{s}_{j}^{(k)}\right) \mid \forall k=1: N_{s}, \forall j \in \Psi_{i}\right\}$, the VIP algorithm can iteratively identify $\widehat{\mathbf{s}}_{i}$, even with nondeterministic measurement precision $\mathrm{w}_{i, j}$. The other nondeterministic parameters (i.e., $\mathbf{U}_{i}$ and $\mathrm{w}_{i, j}$ ) can also be updated with decreasing errors, leading to more accurate network localization. The pseudo-code description of the proposed VIP scheme is presented in Algorithm 1.

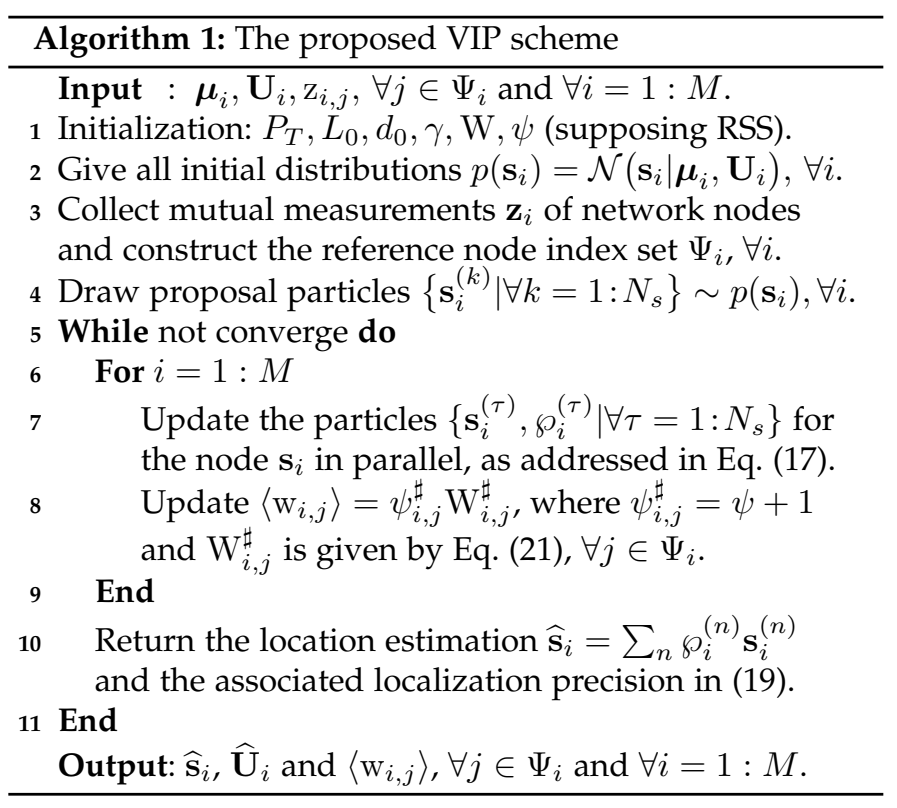

\subsection{Algorithm Characteristics}

In order to clearly present the mechanism of the proposed VIP algorithm, we have the following formulation.

(i) Message Propagation: We can see from Eq. (17) that, in the VIP algorithm, by incorporating $q\left(\mathbf{s}_{j}\right)$ and $q\left(\mathrm{w}_{i, j}\right)$, $\forall j \in \Psi_{i}$, (which converge to $p\left(\mathbf{s}_{j} \mid \mathbf{z}_{i}\right)$ and $p\left(\mathrm{w}_{i, j} \mid \mathbf{z}_{i}\right)$, respectively), the particle set $\left\{\mathbf{s}_{i}^{(k)}, \wp_{i}^{(k)} \mid \forall k=1: N_{s}\right\}$ yields a more and more closer approximation to $p\left(\mathbf{s}_{i} \mid \mathbf{z}_{i}\right)$ by using $q\left(\mathbf{s}_{i}\right)$. As a result, the approximation error of particle set $\left\{\mathbf{s}_{i}^{(k)}, \wp_{i}^{(k)} \mid \forall k=1: N_{s}\right\}$ will become smaller and smaller gradually.

In the framework of VIP algorithm, the estimation of one variable $\boldsymbol{\alpha}_{i}^{m}\left(\forall \boldsymbol{\alpha}_{i}^{m} \in\left\{\boldsymbol{\alpha}_{i}\right\}\right)$ benefits from the variational information of other variables in the associated MB $\mathcal{B}\left(\boldsymbol{\alpha}_{i}^{m}\right)$.

Nevertheless, for the traditional importance samplingbased positioning method (e.g., [20]), the desired particles $\left\{\mathbf{s}_{i}^{(k)}, \wp_{i}^{(k)} \mid \forall k=1: N_{s}\right\}$ only depend on priori $p\left(\mathbf{s}_{j}\right)$ and $p\left(\mathrm{w}_{i, j}\right), \forall j \in \Psi_{i}$. Given the proposal particles $\left\{\mathbf{s}_{i}^{(k)} \mid \forall k=1\right.$ : $\left.N_{s}\right\}$ drawn from $p\left(\mathbf{s}_{i}\right)$, each particle weight is calculated as Eq. (24), where the particle set $\left\{\mathbf{s}_{j}^{(m)}, \wp_{j}^{(m)} \mid \forall m=1: N_{s}\right\}$ approximates the priori $p\left(\mathbf{s}_{j}\right)$, and $\left\{\mathrm{w}_{i, j}^{(n)}, \varphi_{i, j}^{(n)} \mid \forall n=1: N_{s}\right\}$ approximates the priori $p\left(\mathrm{w}_{i, j}\right), \forall j \in \Psi_{i}$. Namely, there is just the priori rather than the posteriori information of $\mathbf{s}_{j}$ and $\mathrm{w}_{i, j}$ propagated into the localization of node $\mathbf{s}_{i}$. Hence, the particle approximation involved in the VIP algorithm is more efficient than that in the traditional importancesampling method.

On the other hand, by incorporating the variational message $\left\{\mathcal{P}_{i}\left(\mathbf{s}_{j}^{(\tau)}\right) \mid \forall \tau=1: N_{s}\right\}$ from reference node $\mathbf{s}_{j}$, $\forall j \in \Psi_{i}$ (which can also incorporate variational message $\left\{\mathcal{P}_{j}\left(\mathbf{s}_{k}^{(t)}\right) \mid \forall t=1: N_{s}\right\}$ from its own reference node $\mathbf{s}_{k}$, $\left.\forall k \in \Psi_{j}\right)$, the node $\mathbf{s}_{i}$ can integrate the localization information from the remote node $\mathbf{s}_{k}, k \notin \Psi_{i}$, that falls out of its reference cluster, as shown in Fig. 2. In such a case, 


$$
\wp_{i}^{(k)} \propto \prod_{j \in \Psi_{i}} \sum_{\substack{m=1: N_{s}, n=1: N_{s}}} \wp_{j}^{(m)} \varphi_{i, j}^{(n)}\left|\mathrm{w}_{i, j}^{(n)}\right|^{\frac{1}{2}} \exp \left(-\frac{1}{2} \mathrm{w}_{i, j}^{(n)}\left(\mathrm{z}_{i, j}-h\left(\mathbf{s}_{i}^{(k)}, \mathbf{s}_{j}^{(m)}\right)\right)^{2}\right) .
$$

the mutual measurement information of all sensor nodes can be globally shared among the whole network. However, the traditional importance-sampling method (e.g., [20]) only incorporates the associated local priori information of $p\left(\mathbf{s}_{j}\right)$ and $p\left(\mathrm{w}_{i, j}\right), \forall j \in \Psi_{i}$, from its reference cluster.

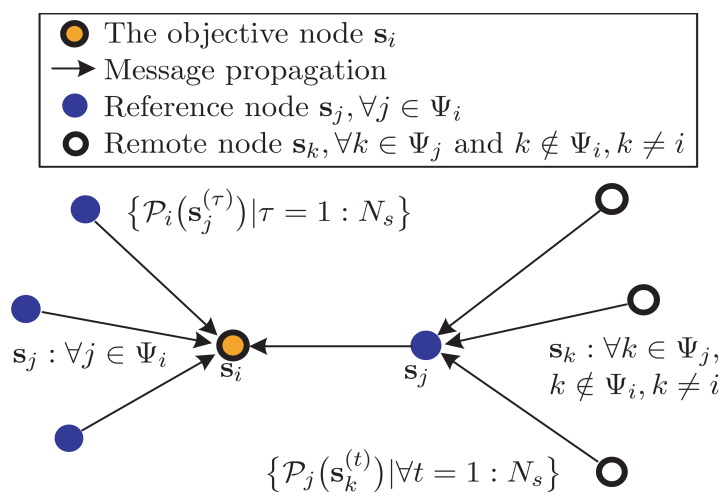

Fig. 2. Illustration of variational message propagation.

Hence, the proposed VIP scheme gains further localization accuracy from both the global localization information propagation among network nodes and the variational message exchange with the associated MB variables.

(ii) Parallel Structure: The calculation steps 7 and 8 of the VIP algorithm can be performed in parallel when iteratively deducing each approximate distribution $q\left(\boldsymbol{\alpha}_{i}^{m}\right)$, $\forall \boldsymbol{\alpha}_{i}^{m} \in\left\{\boldsymbol{\alpha}_{i}\right\}$. Consequently, in the VIP algorithm, the parallel calculations can be performed as follows. (1) All node locations $\mathbf{s}_{i}$ and the measurement precisions $\mathrm{w}_{i, j}, \forall j \in \Psi_{i}$, $\forall i=1: M$, can be deduced in parallel, through exchanging the associated variational information; (11) At each VBI iteration of each node location, all weights $\left\{\wp_{i}^{(\tau)} \mid \forall \tau=1: N_{s}\right\}$ of its variational particle set can also be updated in parallel (see (17)); (111) For the update of a particle weight $\wp_{i}^{(\tau)}$ of a node $\mathbf{s}_{i}$, all variational messages $\left\{\mathcal{P}_{i}\left(\mathbf{s}_{j}^{(\tau)}\right)\right\} \underset{\substack{\forall \tau=1: N_{s} \\ \forall j \in \Psi_{i}}}{\text { propagate }}$ from its reference nodes $\left\{\mathbf{s}_{j}: \forall j \in \Psi_{i}\right\}$ can be calculated in parallel, as shown in Fig. 3 .

(iii) VIP Convergence: Generally, the variational inference estimator converges to a saddle point. If the localization system is unbiased, the VIP-based location estimation can converge to the true location asymptotically. The convergence properties of variational Bayesian approximation method are studied in [49], which states that the variational inference-based unbiased estimation will locally converge to the true value almost surely when the measurement sample size is sufficiently large. In lights of this, the variational inference-based approximation error will approach zero, when the number of variational particles and measurement samples approach infinity. Moreover, the associated localization error is asymptotically Gaussian [49].

On the other hand, in our VIP algorithm, the variational approximation $q\left(\boldsymbol{\alpha}_{i}^{n}\right), \forall \boldsymbol{\alpha}_{i}^{n} \in\left\{\boldsymbol{\alpha}_{i}\right\}$, is chosen to minimize the KLD distance $D_{\mathrm{KL}}\left[q\left(\boldsymbol{\alpha}_{i}\right)|| p\left(\boldsymbol{\alpha}_{i} \mid \mathbf{z}_{i}\right)\right]$ in (10), we then have

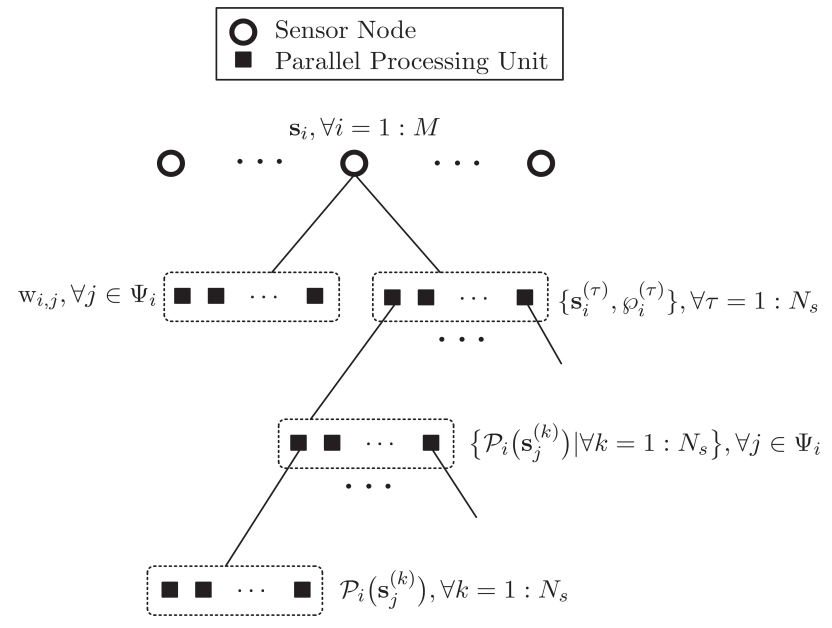

Fig. 3. Illustration of the parallel computation of the VIP algorithm.

that $0 \leq D_{\mathrm{KL}}^{(\ell)}\left[q\left(\boldsymbol{\alpha}_{i}\right) \| p\left(\boldsymbol{\alpha}_{i} \mid \mathbf{z}_{i}\right)\right] \leq D_{\mathrm{KL}}^{(\ell-1)}\left[q\left(\boldsymbol{\alpha}_{i}\right) \| p\left(\boldsymbol{\alpha}_{i} \mid \mathbf{z}_{i}\right)\right]$, where $\bar{D}_{\mathrm{KL}}^{(\ell)}\left[q\left(\boldsymbol{\alpha}_{i}\right) \| p\left(\boldsymbol{\alpha}_{i} \mid \mathbf{z}_{i}\right)\right]$ denotes the associated KLD at the $\ell$ th variational iteration. Hence, for each iteration the KLD is monotonically non-increasing. Once the proposed VIP method reaches a set of fixed distributions $\left\{q\left(\boldsymbol{\alpha}_{i}^{n}\right)\right.$ : $\left.\forall \boldsymbol{\alpha}_{i}^{n} \in\left\{\boldsymbol{\alpha}_{i}\right\}\right\}$, the variational distribution $q\left(\boldsymbol{\alpha}_{i}\right)$ minimizes $D_{\mathrm{KL}}\left[q\left(\boldsymbol{\alpha}_{i}\right) \| p\left(\boldsymbol{\alpha}_{i} \mid \mathbf{z}_{i}\right)\right]$. As a result, the VIP algorithm can make $\left\{q\left(\boldsymbol{\alpha}_{i}^{n}\right): \forall \boldsymbol{\alpha}_{i}^{n} \in\left\{\boldsymbol{\alpha}_{i}\right\}\right\}$ as close as possible to $p\left(\boldsymbol{\alpha}_{i} \mid \mathbf{z}_{i}\right)$, till it finally converges. The VIP algorithm converges typically after 6 iterations.

(iv) Computational Complexity: For the localization of one node, the VIP algorithm's computational complexity scales with $\mathcal{O}\left(M_{i} N_{s}^{2} \mathcal{T}\right)$, where $\mathcal{T}$ is the iteration number such that the VIP algorithm converges. As mentioned earlier, all system variables and variational particles in the VIP algorithm can be calculated in parallel. Hence, in one parallel processing unit, the practical computational overhead is reduced to $\mathcal{O}(\mathcal{T})$, meanwhile $M_{i} N_{s}^{2}$ processing units are required. Although the overall computational cost is equivalent to a centralized strategy, the required processing time can be decreased by $M_{i} N_{s}^{2}$ times if the parallel-computation strategy is employed. The computation complexity of traditional Monte Carlo-based method (e.g., [20]) scales with $\mathcal{O}\left(M_{i} N_{s}^{3}\right)$, as shown in Eq. (24).

\section{Performance Analysis}

In addition to complexity and convergence, there are other localization performance metrics, such as CRLB, robustness, scalability and cost, where the CRLB is commonly used to benchmark the localization precision, which is defined as the inverse of a Fisher Information Matrix (FIM) [50].

In this section, we first present a full FIM associated with the localization system, which holds all statistical inference information of all nondeterministic variables (such as node location variables $\mathbf{s}_{i}, \mathbf{s}_{j}$ and measurement accuracy $\mathrm{w}_{i, j}$ ). 
Based on this result, the node location estimation-based CRLB is then obtained to evaluate the VIP-based localization performance. Finally, we perform a CRLB analysis and find the asymptotic performance limits over the size of reference node location error and the number of reference nodes.

\subsection{Full FIM}

Consider the localization of sensor node $\mathbf{s}_{i}$, whose complete variable (that incorporates all the associated nondeterministic variables) is denoted as $\boldsymbol{\alpha}_{i}$ (see its definition in Section 3.1). The full FIM associated with the Bayesian estimation (BE) of complete variable $\boldsymbol{\alpha}_{i}$ is expressed as [50],

$$
\begin{aligned}
& \boldsymbol{J}_{\mathrm{BE}}^{\boldsymbol{\alpha}_{i}}=-\mathbb{E}_{\boldsymbol{\alpha}_{i}, \mathbf{z}_{i}}\left\{\nabla_{\boldsymbol{\alpha}_{i}, \boldsymbol{\alpha}_{i}^{\top}} \ln p\left(\boldsymbol{\alpha}_{i} \mid \mathbf{z}_{i}\right)\right\}, \\
& =\underbrace{-\mathbb{E}_{\mathbf{z}_{i}, \boldsymbol{\alpha}_{i}}\left\{\nabla_{\boldsymbol{\alpha}_{i}, \boldsymbol{\alpha}_{i}^{\top}} \ln p\left(\mathbf{z}_{i} \mid \boldsymbol{\alpha}_{i}\right)\right\}}_{\boldsymbol{J}_{\mathrm{MLE}}^{\boldsymbol{\alpha}_{i}}} \underbrace{-E_{\boldsymbol{\alpha}_{i}}\left\{\nabla_{\boldsymbol{\alpha}_{i}, \boldsymbol{\alpha}_{i}^{\top}} \ln p\left(\boldsymbol{\alpha}_{i}\right)\right\}}_{\boldsymbol{J}_{\mathrm{P}}^{\boldsymbol{\alpha}_{i}}},
\end{aligned}
$$

where $\nabla_{\boldsymbol{\alpha}_{i}, \boldsymbol{\alpha}_{i}^{\top}}(\bullet)$ denotes a second order derivative with respect to $\boldsymbol{\alpha}_{i}, \mathbb{E}_{\mathbf{z}_{i}, \boldsymbol{\alpha}_{i}}\{\bullet\}$ denotes the expectation with respect to distribution $p\left(\mathbf{z}_{i}, \boldsymbol{\alpha}_{i}\right), \boldsymbol{J}_{\mathrm{MLE}}^{\boldsymbol{\alpha}_{i}}$ is the maximum likelihood estimation (MLE)-based FIM, and $\boldsymbol{J}_{\mathrm{P}}^{\boldsymbol{\alpha}_{i}}$ is the priori FIM.

Assuming the variables are priori independent, the BEbased FIM can be structured as Eq. (26), where $M_{i}$ reference nodes in $\Phi_{i}$ are successively labeled by $\mathbf{s}_{1}, \ldots, \mathbf{s}_{M_{i}}$. The MLE-based FIM $\mathcal{I}_{\mathrm{MLE}}^{\boldsymbol{\alpha}_{i}^{m}, \boldsymbol{\alpha}_{i}^{n}}$ of $\boldsymbol{\alpha}_{i}^{m}, \boldsymbol{\alpha}_{i}^{n}, \forall \boldsymbol{\alpha}_{i}^{m}, \boldsymbol{\alpha}_{i}^{n} \in\left\{\boldsymbol{\alpha}_{i}\right\}$, and the priori FIM $\mathcal{I}_{\mathrm{P}}^{\boldsymbol{\alpha}_{i}^{n}}$ of variable $\boldsymbol{\alpha}_{i}^{m}$ are defined as

$$
\begin{aligned}
\mathcal{I}_{\mathrm{MLE}}^{\boldsymbol{\alpha}_{i}^{m}, \boldsymbol{\alpha}_{i}^{n}} & =-\mathbb{E}_{\mathbf{z}_{i}, \boldsymbol{\alpha}_{i}}\left\{\nabla_{\boldsymbol{\alpha}_{i}^{m}, \boldsymbol{\alpha}_{i}^{n} \top} \ln p\left(\mathbf{z}_{i} \mid \boldsymbol{\alpha}_{i}\right)\right\}, \\
\mathcal{I}_{\mathrm{P}}^{\boldsymbol{\alpha}_{i}^{m}} & =-\mathbb{E}_{\boldsymbol{\alpha}_{i}}\left\{\nabla_{\boldsymbol{\alpha}_{i}^{m}, \boldsymbol{\alpha}_{i}^{m} \top} \ln p\left(\boldsymbol{\alpha}_{i}^{m}\right)\right\},
\end{aligned}
$$

which are specified as (see APPENDIX C, D and E)

$$
\begin{aligned}
\mathcal{I}_{\mathrm{MLE}}^{\mathbf{s}_{i}, \mathbf{s}_{i}} & =\sum_{j \in \Psi_{i}} \eta \mathbf{A}_{i, j}, \\
\mathcal{I}_{\mathrm{P}}^{\mathbf{s}_{i}} & =\mathbf{U}_{i}, \\
\mathcal{I}_{\mathrm{MLE}}^{\mathbf{s}_{i}, \mathbf{s}_{j}} & =-\eta \mathbf{A}_{i, j}, \forall j \in \Psi_{i}, \\
\mathcal{I}_{\mathrm{MLE}}^{\mathbf{s}_{i}, \mathbf{w}_{i, j}} & =\mathbf{0}_{\mathfrak{D} \times 1}, \forall j \in \Psi_{i}, \\
\mathcal{I}_{\mathrm{MLE}}^{\mathbf{s}_{j}, \mathbf{s}_{j}} & =\eta \mathbf{A}_{i, j}, \forall j \in \Psi_{i}, \\
\mathcal{I}_{\mathrm{P}}^{\mathbf{s}_{j}} & =\mathbf{U}_{j}, \forall j \in \Psi_{i}, \\
\mathcal{I}_{\mathrm{MLE}}^{\mathbf{s}_{j}, \mathbf{s}_{k}} & =\mathbf{0}_{(\mathfrak{D} \times \mathfrak{D})}, \forall j, k \in \Psi_{i} \text { and } j \neq k,
\end{aligned}
$$

and other information vector $\mathcal{I}_{\mathrm{MLE}}^{\mathrm{s}_{k}, \mathrm{~W}_{i, j}}, \forall j, k \neq i$ are not included here, since they will multiply with all-zero information metric $\mathcal{I}_{\mathrm{MLE}}^{\mathrm{S}_{i}, \mathrm{w} i, j}$ (see Eq. (32) and (40)). In addition, $\eta$ stands for the measurement precision expectation,

$$
\eta=\mathrm{W} \psi,
$$

and $\mathbf{A}_{i, j}$ denotes the measurement resolution factor, which is specified as (based on the specific measurement choices)

$$
\begin{aligned}
\mathbf{A}_{i, j}= & \mathbb{E}_{\mathbf{s}_{i}, \mathbf{s}_{j}}\left\{\nabla_{\mathbf{s}_{i}} h\left(\mathbf{s}_{i}, \mathbf{s}_{j}\right) \nabla_{\mathbf{s}_{j}^{\top}} h\left(\mathbf{s}_{i}, \mathbf{s}_{j}\right)\right\} \\
= & \left\{\begin{array}{l}
\left(\frac{10 \gamma}{\ln 10}\right)^{2} \mathbb{E}_{\mathbf{s}_{i}, \mathbf{s}_{j}}\left\{\frac{\left(\mathbf{s}_{i}-\mathbf{s}_{j}\right)\left(\mathbf{s}_{i}-\mathbf{s}_{j}\right)^{\top}}{\left\|\mathbf{s}_{i}-\mathbf{s}_{j}\right\|_{2}^{4}}\right\}, \\
\mathbb{E}_{\mathbf{s}_{i}, \mathbf{s}_{j}}\left\{\frac{\left(\mathbf{s}_{i}-\mathbf{s}_{j}\right)\left(\mathbf{s}_{i}-\mathbf{s}_{j}\right)^{\top}}{\left\|\mathbf{s}_{i}-\mathbf{s}_{j}\right\|_{2}^{2}}\right\}, \\
\left(\frac{180}{\pi}\right)^{2} \mathbb{E}_{\mathbf{s}_{i}, \mathbf{s}_{j}}\left\{\frac{\mathbf{v}_{i, j} \mathbf{v}_{i, j}^{\top}}{\left\|\mathbf{s}_{i}-\mathbf{s}_{j}\right\|_{2}^{4}}\right\},
\end{array}\right.
\end{aligned}
$$

where the vector $\mathbf{v}_{i, j}$ in the AOA case (supposing the dimensions of location space are $\mathfrak{D}=2$ ) is defined as

$$
\mathbf{v}_{i, j}=\left[\begin{array}{c}
{\left[\mathbf{s}_{j}\right]_{2}-\left[\mathbf{s}_{i}\right]_{2}} \\
{\left[\mathbf{s}_{i}\right]_{1}-\left[\mathbf{s}_{j}\right]_{1}}
\end{array}\right] .
$$

The measurement resolution factor $\mathbf{A}_{i, j}$ indicates the capability that the localization algorithm precisely recognizes the location difference, given a certain measurement change.

Moreover, by letting $\mathcal{I}_{\mathrm{BE}}^{\mathrm{s}_{i}, \mathbf{s}_{i}}=\mathcal{I}_{\mathrm{MLE}}^{\mathrm{s}_{i}, \mathbf{s}_{i}}+\mathcal{I}_{\mathrm{P}}^{\mathrm{s}_{i}}$, the full BEbased FIM $\boldsymbol{J}_{\mathrm{BE}}^{\alpha_{i}}$ can be partitioned as

$$
\boldsymbol{J}_{\mathrm{BE}}^{\boldsymbol{\alpha}_{i}}=\left[\begin{array}{cc}
\mathcal{I}_{\mathrm{BE}}^{\mathbf{s}_{i}, \mathbf{s}_{i}} & \left(\boldsymbol{\Phi}_{\mathrm{BE}}^{\mathbf{s}_{i}}\right)^{\top} \\
\boldsymbol{\Phi}_{\mathrm{BE}}^{\mathbf{s}_{i}} & \boldsymbol{\mathcal { R }}_{\mathrm{BE}}^{\mathbf{s}_{i}}
\end{array}\right] .
$$

\subsection{VIP-associated FIM}

We now focus on the estimation of $\mathbf{s}_{i}$ over nuisance parameters $\mathbf{s}_{j}$ and $\left\{\mathrm{w}_{i, j} \mid \forall j \in \Psi_{i}\right\}$. The overall Bayesian information of the objective node localization can be characterized by its BE-based full FIM $\boldsymbol{J}_{\mathrm{BE}}^{\boldsymbol{\alpha}_{i}}$ in (26), and the equivalent FIM $\boldsymbol{J}_{\mathrm{BE}}^{\mathbf{s}_{i}}$ associated with $\mathbf{s}_{i}$ retains all necessary information for localization, in terms of $\left[\left(\boldsymbol{J}_{\mathrm{BE}}^{\boldsymbol{\alpha}_{i}}\right)^{-1}\right]_{[1: \mathcal{D}, 1: \mathcal{D}]}=\left(\boldsymbol{J}_{\mathrm{BE}}^{\mathbf{S}_{i}}\right)^{-1}[11]$.

Based on the matrix partition in Eq. (39), the equivalent Bayesian FIM $\boldsymbol{J}_{\mathrm{BE}}^{\mathbf{s}_{i}}$ associated with localization of $\mathbf{s}_{i}$ can be derived by using Schur's complement as

$$
\boldsymbol{J}_{\mathrm{BE}}^{\mathbf{s}_{i}}=\mathcal{I}_{\mathrm{BE}}^{\mathbf{s}_{i}, \mathbf{s}_{i}}-\left(\boldsymbol{\Phi}_{\mathrm{BE}}^{\mathbf{s}_{i}}\right)^{\top}\left(\mathcal{R}_{\mathrm{BE}}^{\mathbf{s}_{i}}\right)^{-1} \boldsymbol{\Phi}_{\mathrm{BE}}^{\mathbf{s}_{i}}
$$

Based on (29)-(35), we have (see APPENDIX F)

$$
\begin{aligned}
\boldsymbol{J}_{\mathrm{BE}}^{\mathbf{s}_{i}} & =\mathbf{U}_{i}+\sum_{j \in \Psi_{i}} \eta \mathbf{A}_{i, j}-\sum_{j \in \Psi_{i}} \eta^{2} \mathbf{A}_{i, j}^{\top}\left(\eta \mathbf{A}_{i, j}+\mathbf{U}_{j}\right)^{-1} \mathbf{A}_{i, j} \\
& =\sum_{j \in \Psi_{i}} \underbrace{\left(\left(\eta \mathbf{A}_{i, j}\right)^{-1}+\mathbf{U}_{j}^{-1}\right)^{-1}}_{\mathcal{H}_{i, j}}+\mathbf{U}_{i},
\end{aligned}
$$

where $\mathcal{H}_{i, j}$ is defined as the equivalent measurement information from $\mathbf{s}_{j}$ accounting for its location uncertainty, while $\eta \mathbf{A}_{i, j}$ corresponds to the crude measurement information without considering the reference location uncertainty.

Based on Eq. (41), the localization performance depends on the measurement size, the independent reference source size $\left|\Psi_{i}\right|$, the priori accuracy $\mathbf{U}_{i}$ and $\mathbf{U}_{j}$, the measurement resolution $\mathbf{A}_{i, j}$ and the measurement precision. In addition, considering the randomness of measurement precision, the final VIP error bound only depends on the measurement precision's expectation, as shown in Eq. (41). Even so, when one assumes the measurement precision of all nodes is fixed at its expectation (if its expectation could be known beforehand somehow), the corresponding localization method will lose certain performance, just as the traditional localization methods did. Namely, capturing the statistics of measurement precision will gain further performance.

As shown in Eq. (41), each crude measurement information $\eta \mathbf{A}_{i, j}$ (neglecting the reference node location error) gathers measurement precision expectation $\eta$ and measurement resolution $\mathbf{A}_{i, j}$. The measurement resolution $\mathbf{A}_{i, j}$ indicates the discrimination ability of the corresponding localization method, while the inverse of measurement precision expectation $\eta$ implies how seriously the measurement noises blur the location estimation.

In addition, due to the reference node location errors $\left\{\mathbf{U}_{j}^{-1}: \forall j \in \Psi_{i}\right\}$, the final localization accuracy is reduced 


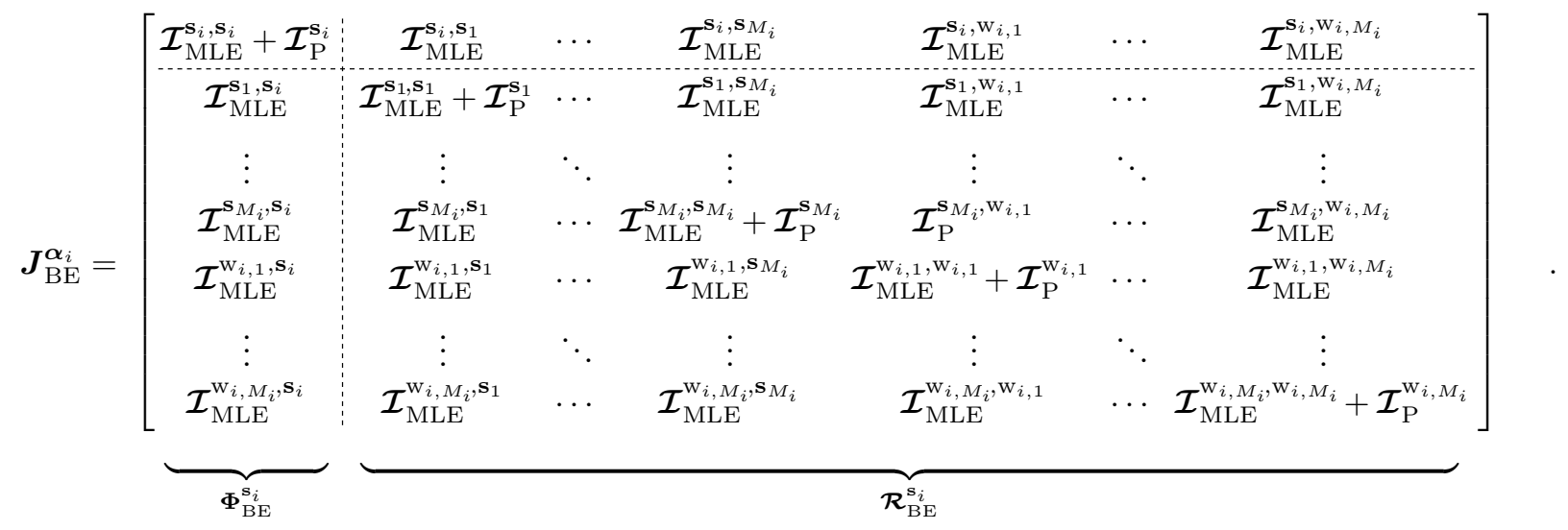

with $\sum_{j \in \Psi_{i}} \eta^{2} \mathbf{A}_{i, j}^{\top}\left(\eta \mathbf{A}_{i, j}+\mathbf{U}_{j}\right)^{-1} \mathbf{A}_{i, j}$. Moreover, measurement information from each reference node is degraded from $\eta \mathbf{A}_{i, j}$ to $\mathcal{H}_{i, j}$. Thereafter, all these weakened measurement information $\sum_{j \in \Psi_{i}} \mathcal{H}_{i, j}$ collaborates with the priori accuracy $\mathbf{U}_{i}$ to produce the final localization accuracy information $\boldsymbol{J}_{\mathrm{BE}}^{\mathbf{s}_{i}}$.

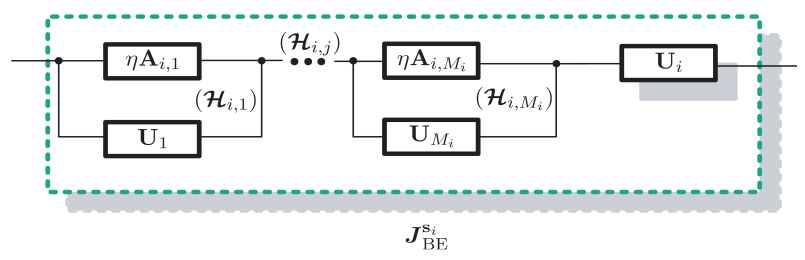

Fig. 4. Illustration of the spatial propagation of localization information.

Furthermore, we can see that all the involved localization information $\left(\left\{\eta \mathbf{A}_{i, j}, \mathbf{U}_{j}: \forall j \in \Psi_{i}\right\}\right.$ and $\left.\mathbf{U}_{i}\right)$ propagates spatially like the Ohm's Law in circuit theory. As shown in Fig. 4, for the localization of node $\mathbf{s}_{i}$, the crude measurement information $\eta \mathbf{A}_{i, j}$ and location precision $\mathbf{U}_{j}$ of one reference node $\mathbf{s}_{j}$ can be deemed as resistances connected in parallel, forming the equivalent measurement information $\mathcal{H}_{i, j}$ (i.e., $R_{1}=\left(R_{1,1}^{-1}+R_{1,2}^{-1}\right)^{-1}$, where $R_{1}$ stands for the equivalent resistance of two parallel-connected resistances $R_{1,1}$ and $R_{1,2}$ ); these equivalent measurement information $\left\{\mathcal{H}_{i, j}: \forall j \in \Psi_{i}\right\}$ from all reference nodes and its priori location precision $\mathbf{U}_{i}$ propagate like the resistances connected in series (resistance summation), forming the final localization information $\boldsymbol{J}_{\mathrm{BE}}^{\mathbf{s}_{i}}$ of node $\mathbf{s}_{i}$.

\subsection{CRLB Analysis}

On the other hand, the mean square error of the VIP-based localization is lower bounded by its CRLB $\mathfrak{B}_{\mathrm{BE}}^{\mathbf{s}_{i}}$ as follows

$$
\begin{aligned}
\mathbb{E}\left\{\left\|\widehat{\mathbf{s}}_{i}-\mathbf{s}_{i}\right\|_{2}^{2}\right\} & \geq \operatorname{tr}\left(\boldsymbol{B}_{\mathrm{BE}}^{\mathbf{s}_{i}}\right), \\
\mathfrak{B}_{\mathrm{BE}}^{\mathbf{s}_{i}} & =\left(\boldsymbol{J}_{\mathrm{BE}}^{\mathbf{s}_{i}}\right)^{-1},
\end{aligned}
$$

where $\operatorname{tr}(\bullet)$ denotes the matrix trace.

Considering node location uncertainties in the cooperative localization, a natural question arises: Does a reference node contribute to localizing the objective node even though its location is inaccurate? By introducing $\mathfrak{B}_{\mathrm{BE}}^{\mathbf{s}_{i}}\left(M_{i}\right)$ to denote the CRLB of node $\mathbf{s}_{i}$, associated with $M_{i}$ reference nodes, we have the following proposition.
Proposition 1. Assume the current reference cluster size is $M_{i}$, and give one more reference sensor $\mathbf{s}_{M_{i}+1}$ with location precision $\mathbf{U}_{M_{i}+1}$, then the CRLB satisfies

$$
\mathfrak{B}_{\mathrm{BE}}^{\mathbf{s}_{i}}\left(M_{i}+1\right) \preceq \mathfrak{B}_{\mathrm{BE}}^{\mathbf{s}_{i}}\left(M_{i}\right) .
$$

Proof. Since both $\mathbf{U}_{M_{i}+1}$ and $\eta \mathbf{A}_{i, M_{i}+1}$ are positive, the above inequality can be directly derived from Eq. (41).

Proposition 1 implies that, even if the location precision of a node is very poor, incorporating this node into reference cluster can still statistically improve the localization accuracy. Namely, there still exists useful information from those location-precision-limited nodes for network localization.

We define $\mathbf{U}_{i}^{\prime} \doteq \max \left\{\mathbf{U}_{j}:\left\|\mathbf{U}_{j}\right\|_{2}, \forall j \in \Psi_{i}\right\}$ and $\mathbf{U}_{i}^{\prime \prime}=$ $\min \left\{\mathbf{U}_{j}:\left\|\mathbf{U}_{j}\right\|_{2}, \forall j \in \Psi_{i}\right\}$, in which $\|\bullet\|_{2}$ denotes the Hilbert-Schmidt norm for squared matrix. We have

$$
\begin{aligned}
\lim _{\mathbf{U}_{i}^{\prime} \rightarrow \mathbf{0}} \mathfrak{B}_{\mathrm{BE}}^{\mathbf{s}_{i}} & =\mathbf{U}_{i}^{-1}, \\
\lim _{\mathbf{U}^{\prime \prime} \rightarrow \infty} \boldsymbol{B}_{\mathrm{BE}}^{\mathbf{s}_{i}} & =(\underbrace{\sum_{j \in \Psi_{i}} \eta \mathbf{A}_{i, j}+\mathbf{U}_{i}}_{\boldsymbol{J}_{\infty}^{\mathbf{s}_{i}}})^{-1},
\end{aligned}
$$

where $\mathbf{U}_{i}^{\prime \prime} \rightarrow \infty$ denotes $\left\|\mathbf{U}_{i}^{\prime \prime}\right\|_{2}-\|\mathbf{H}\|_{2}>0, \forall \mathbf{H} \in \mathbb{R}^{\mathfrak{D} \times \mathfrak{D}}$.

Based on Proposition 1 we further know that, the associated CRLB reduces with the increase of reference cluster size, despite node location errors. Another question arises. What is the limit of CRLB with infinitely large reference cluster?

Proposition 2. When all network nodes are randomly and uniformly distributed inside the deployment area, the localization accuracy scales linearly with the reference cluster sizes (keeping a fixed sensing range $r_{s}$ ), namely,

$$
\mathcal{J}_{\mathrm{BE}}^{\mathbf{s}_{i}}=M_{i} \mathcal{H}+\mathbf{U}_{i}
$$

where $\mathcal{H}=\left((\eta \boldsymbol{\Lambda})^{-1}+\overline{\mathbf{U}}^{-1}\right)^{-1}$ denotes the expectation of equivalent measurement information $\mathcal{H}_{i, j}$, in a long-term averaging sense. $\boldsymbol{\Lambda}$ and $\overline{\mathbf{U}}$ stand for the expectation of $\mathbf{A}_{i, j}$ and $\mathbf{U}_{j}$, respectively.

Proof. Please see the proof in APPENDIX G. 
We can see from Eq. (47) that

$$
\begin{aligned}
\left(\operatorname{tr}\left(\mathfrak{B}_{\mathrm{BE}}^{\mathbf{s}_{i}}\right)\right)^{\frac{1}{2}} & =\left(\operatorname{tr}\left(\left(M_{i} \mathcal{H}+\mathbf{U}_{i}\right)^{-1}\right)\right)^{\frac{1}{2}}, \\
\lim _{M_{i} \rightarrow \infty}\left(\operatorname{tr}\left(\mathfrak{B}_{\mathrm{BE}}^{\mathbf{s}_{i}}\right)\right)^{\frac{1}{2}} & =0 .
\end{aligned}
$$

Namely, although all node locations are not accurate, the positioning error (in terms of root mean squared error) tends to be arbitrarily small, at the rate of $\mathcal{O}\left((\sqrt{M})^{-1}\right)$, when the reference cluster size is sufficiently large.

\section{Numerical Simulation}

\subsection{Simulation Settings}

Numerical simulation results are presented herein to show various performance of the VIP-based localization.

We consider a static WSN deployed in an $R[\mathrm{~m}] \times R[\mathrm{~m}]$ squared area, where $R$ denotes the width of the deployment area. We assume there are only inaccurate sensor nodes (no accurate anchor node) uniformly deployed inside the area.

Firstly, in order to examine the impacts of dependent factors, e.g., the shadow fading, reference cluster size $M_{i}$ and reference node location errors on the VIP performance, Scenarios A1-A3 in TABLE 1 are simulated, respectively, where only RSS measurement is used (assuming $\gamma=3$ and $\phi=50)$. The conclusions obtained can be applied to the TOA and AOA-based localization.

TABLE 1

Scenarios A1-A3 (RSS only)

\begin{tabular}{cccc}
\hline & $\mathrm{A} 1$ & $\mathrm{~A} 2$ & $\mathrm{~A} 3$ \\
\hline$R$ & 20 & 20 & 20 \\
$\mathbf{U}_{j}: \forall j \in \Psi_{i}$ & {$\left[10^{-2} \mathbf{I}, 10^{4} \mathbf{I}\right]^{\dagger}$} & random $^{\ddagger}$ & random \\
$\mathbf{U}_{i}$ & $\mathbf{0}$ & $\mathbf{0}$ & $1 / 10 \mathbf{I}$ \\
$\mathrm{W}$ & $1 / 1500$ & $5 * 10^{-4}: 10^{-3}$ & $1 / 1500$ \\
$\psi$ & 80 & $60: 100$ & 80 \\
$M_{i}$ & 6 & 6 & $3: 100$ \\
\hline
\end{tabular}

${ }^{\dagger}$ Here I represents the identity matrix.

$¥$ The precision of reference node location is assumed to be $\mathbf{U}_{j} \sim \mathcal{W}\left(\mathbf{U}_{j} \mid 1 / 100 \mathbf{I}, 10\right), \forall j \in \Psi_{i}, i=1: M$.

Secondly, Scenario B in TABLE 2 is considered to demonstrate the convergence properties of the VIP scheme, where the measurement is specified as RSS data as well.

TABLE 2

Scenarios B and C

\begin{tabular}{cc}
\hline B (RSS) & $\mathrm{W}=1 / 300: 1 / 15, \psi=8$ \\
C (RSS, TOA, AOA) & $\mathrm{W}=1 / 1500, \psi=80$ \\
\hline
\end{tabular}

In Scenarios $B$ and $C$, we assume the precision of initial node location to be $\mathbf{U}_{i} \sim \mathcal{W}\left(\mathbf{U}_{i} \mid 1 / 100 \mathbf{I}, 10\right)$, $\forall i=1: 200$, and assume $R=100, r_{s}=20$.

Thirdly, Scenario C in TABLE 2 (where RSS, TOA and AOA measurement data are considered respectively) is simulated to compare the proposed VIP algorithm with other localization algorithms, including importance samplingbased positioning (ISP) [20], mulihop-aided particle swar$\mathrm{m}$ optimization (PSO)-based localization [51], weighted least square-based positioning (WLSP) [52], expectationmaximisation-based positioning (EMP) [21], second-order cone programming (SOCP) method [53], semi-definite programming (SDP)-based approach [54], adaptive simulated annealing (ASA)-assisted maximum likelihood localization [55], auxiliary variables-assisted total least square-based positioning (TLSP) [56].

In Scenario $\mathrm{C}$, the localization algorithms utilized in different cases of RSS, TOA and AOA measurements are listed in TABLE 3. In the case of AOA, we assume the direction of antenna main lobe to be $\varphi_{i}=90^{\circ}, \forall i=1: 200$.

TABLE 3

Localization Algorithm Toolbox in Scenario C

\begin{tabular}{cc}
\hline RSS & ISP [20], PSO [51], EMP [21], SOCP [53] \\
WLSP [52] \\
TOA & ISP [20], WLSP [52], EMP [21], SDP [54] \\
AOA & ISP [20], WLSP [52], ASA [55], TLSP [56] \\
\hline
\end{tabular}

It is assumed that the number of particles employed in VIP, ISP and PSO methods is $N_{s}=50$. A total of $10^{3}$ simulations are performed. We use the root mean squared error (RMSE) metric to assess the localization performance, which is defined as

$$
\operatorname{RMSE}=\left(\mathbb{E}\left\{\left\|\widehat{\mathbf{s}}_{i}-\mathbf{s}_{i}\right\|_{2}^{2}\right\}\right)^{\frac{1}{2}} .
$$

\subsection{Influence of Various Dependent Factors}

In this part, the asymptotic localization RMSEs over reference node location precision, shadow fading and the number of reference node number, respectively, are presented to reveal their effects on the proposed VIP-based localization performance.

(i) Reference Node Location Precision $\mathbf{U}_{j}$ : In order to evaluate the VIP performance over different levels of reference node location uncertainties, Scenario A1 is simulated, where we assume the reference node location precision $\mathbf{U}_{j}$, $\forall j \in \Psi_{i}$, varies from $10^{-2} \mathbf{I}$ to $10^{4} \mathbf{I}$, whereas the objective node location precision is fixed at $\mathbf{U}_{i}=1 / 10 \mathbf{I}$. Other simulation settings are given in TABLE 1.

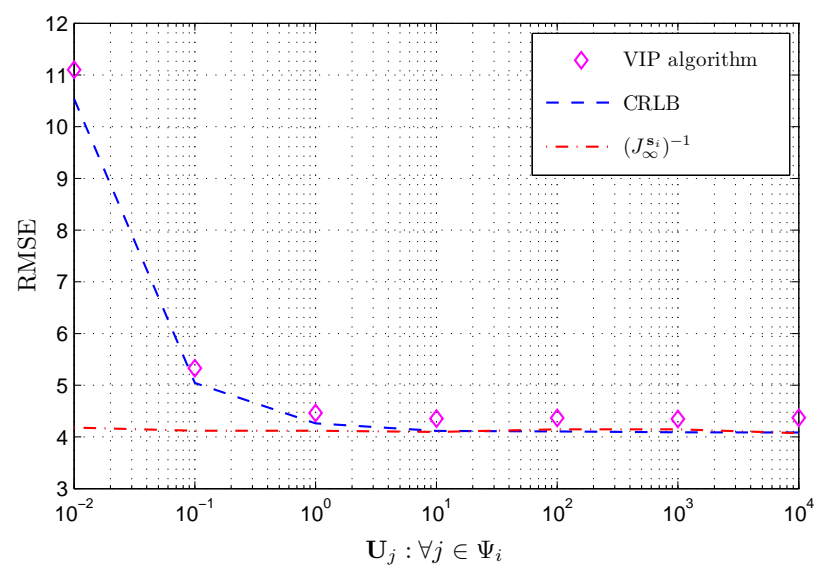

Fig. 5. The RMSEs over different reference node location precision $\mathbf{U}_{j}$ (Scenario A1).

Fig. 5 shows the obtained RMSE v.s. reference node location precision. The dashed curve and dash-dot curve stand for the CRLB and its asymptotic limit $\left(\boldsymbol{J}_{\infty}^{\mathbf{s}_{i}}\right)^{-1}$ (see Eq. (46)), respectively. It is shown that, with the increase in 
the reference node location precision, the obtained RMSE monotonically decreases.

Due to the mechanism to handle the nondeterministic measurement accuracy and reference node errors, the proposed VIP algorithm can get close to its CRLB. However, there is also a little gap from its CRLB, in particular with large reference node location errors. This gap mainly comes from (1) the inevitable approximation error of its variational particles and (11) the inherent gap due to the possible bias, nonlinear or non-Gaussian characteristics of the RSS-based location estimator.

Moreover, the VIP-based localization error and its CRLB reach, at best, the lower limit $\left(\boldsymbol{J}_{\infty}^{\mathbf{s}_{i}}\right)^{-1}$, when the reference location precisions become sufficiently large.

(ii) Fading Parameters: The scale parameter $\mathrm{W}$ and the associated DoF $\psi$ jointly indicate how randomly the measurement noise varies. In order to assess the performance of the proposed VIP algorithm over different levels of shadow fading, Scenario A2 is considered in this simulation. The corresponding simulation settings are given in TABLE 1 .

The achieved RMSEs of the VIP algorithm and its CRLB over different deep-shadow-fading environments are shown in Fig. 6. One can see that the VIP error is close to its CRLB. When the scale $\mathrm{W}$ and the DoF $\psi$ increases, the uncertainty of measurement precision decreases, which leads to the reduced RMSE.

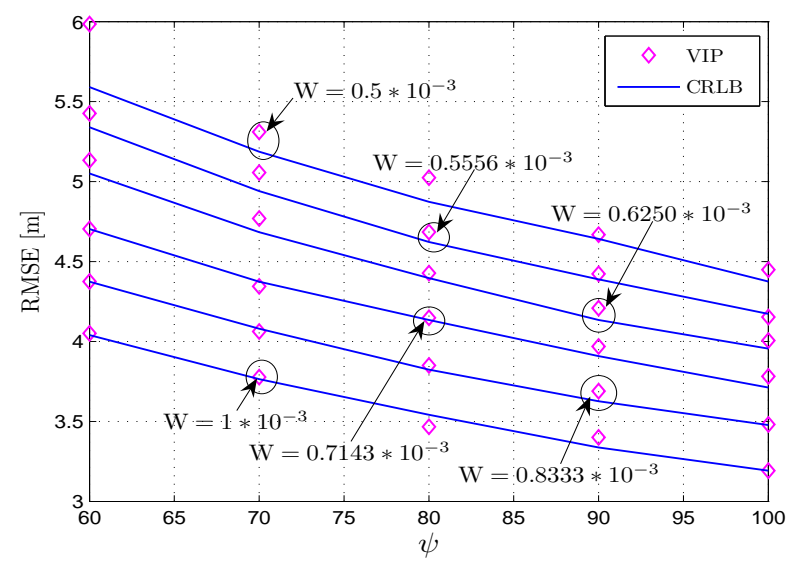

Fig. 6. The achieved RMSEs over different levels of shadowing fading (Scenario A2).

On the other hand, TABLE 4 presents the VIP errors and its CRLB over different shadow fading, wherein the measurement precision expectation $\mathrm{W} \psi$ is fixed, while $\psi$ and W vary. As shown in TABLE 4, the VIP errors are almost invariant in different scenarios, wherein the expectation is set to be $\mathrm{W} \psi=80 / 1500$ while $\psi=60: 100$. Moreover, the same conclusion holds for its CRLB in this case, i.e., the VIP scheme and its CRLB are only dependent on the expectation of measurement precision.

(iii) Reference Cluster Size $M_{i}$ : In this simulation, we consider Scenario A3, where $M_{i}$ is assumed to range from 3 to 100 (while keeping the sensing range $r_{s}=20$ ), to evaluate its impact on network localization. The simulation results are shown in Fig. 7. Other simulation settings are given in TABLE 1.

We can see from Fig. 7(a) that, the localization information $\mathcal{J}_{\mathrm{BE}}^{\mathrm{s}_{i}}$ scales linearly with the reference cluster size $M_{i}$,
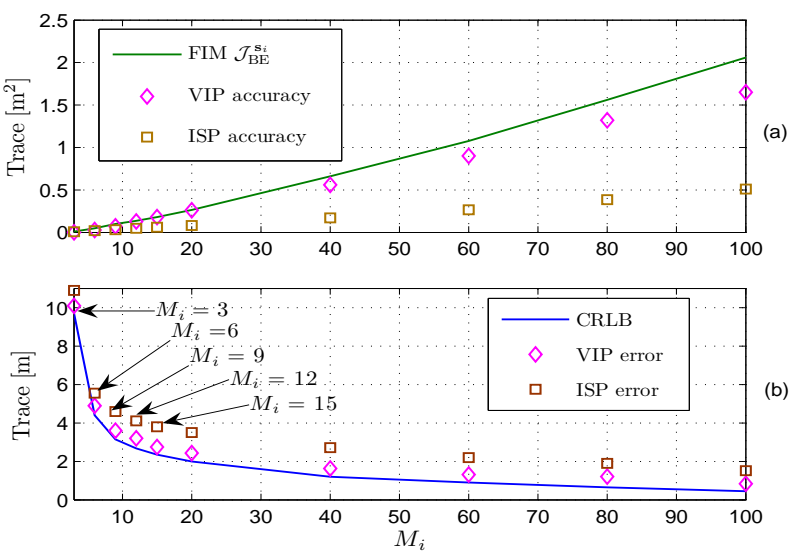

Fig. 7. Localization performance over different reference cluster size $M_{i}$ (Scenario A3). In subfigure (a), we use the matrix trace as a performance metric to assess the localization accuracy and its FIM.

which complies with Proposition 2. Hence, we can expect $\mathfrak{B}_{\mathrm{BE}}^{\mathbf{s}_{i}} \rightarrow \mathbf{0}$ as $M_{i} \rightarrow \infty$. The ISP scheme does not reach the CRLB, due to its limited particle representation efficiency.

\subsection{Convergence of VIP}

According to the framework of the VIP scheme, the node location $\mathbf{s}_{i}$, location precision $\mathbf{U}_{i}$, and the measurement precision $\mathrm{w}_{i, j}$ can be calibrated iteratively at the same time, through propagating the variational message.

In this simulation, Scenario B in TABLE 2 is considered to examine the convergence property of VIP algorithm, in terms of both the estimation error of node location $\widehat{\mathbf{s}}_{i}$ and the calibration error of nondeterministic measurement precision $\widehat{\mathrm{w}}_{i, j}$. The convergence behavior of VIP localization error and measurement precision calibration error over different levels of shadowing are shown in Figs. 8(a) and (b), respectively. The associated simulation settings are given in TABLE 2.
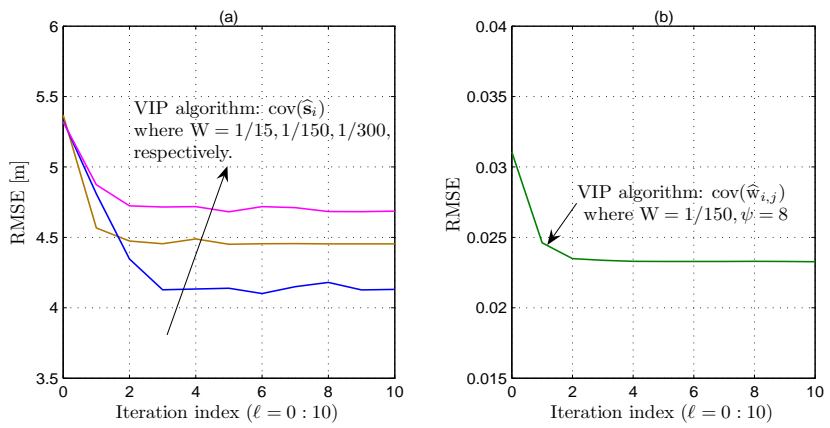

Fig. 8. Convergence of the VIP algorithm (Scenario B).

As shown in Fig. 8(a), the VIP-based localization error can converge to a lower value, which benefits from the global propagation of variational message, at each VIP iteration (see Eq. (17)). A similar convergence behaviour can be found for calibration error of $\widehat{\mathrm{w}}_{i, j}$ in Fig. 8(b). Hence, under the VIP framework, the nondeterministic parameters $\left(\mathrm{w}_{i, j}\right.$ and $\left.\mathbf{U}_{i}\right)$ can be iteratively calibrated with decreasing errors from the given network measurements, which yields a more accurate network localization. 
TABLE 4

Achieved RMSEs with Fixed Expectation of Measurement Precision

\begin{tabular}{c|ccccc}
\hline$(\psi, \mathrm{W})$ & $(60,2 / 2250)$ & $(70,4 / 5250)$ & $(80,1 / 1500)$ & $(90,4 / 6750)$ & $(100,1 / 1875)$ \\
\hline VIP & 5.0332 & 5.0696 & 5.1021 & 5.1315 & 5.0968 \\
CRLB & 4.8952 & 4.8527 & 4.9163 & 4.8875 & 4.9552 \\
\hline
\end{tabular}

Note that, all the achieved RMSEs are in meter.

TABLE 5

The Achieved RMSEs of Different Methods (Scenario C)

\begin{tabular}{rccc}
\hline & RSS & TOA & AOA \\
\hline Proposed VIP & $(4.03,3.13)^{\dagger}$ & $(4.05,3.46)$ & $(3.41,2.32)$ \\
ISP [20] & $(4.54,3.13)$ & $(4.33,3.46)$ & $(3.73,2.32)$ \\
EMP [21] & $\star$ & $(4.31,3.46)$ & $\star$ \\
PSO [51] & $(4.69,3.13)$ & $\star$ & $\star$ \\
WLSP [52] & $(5.34,3.13)$ & $(5.35,3.46)$ & $(5.31,2.32)$ \\
SOCP [53] & $(4.79,3.13)$ & $\star$ & $\star$ \\
SDP [54] & $\star$ & $(4.91,3.46)$ & $\star$ \\
ASA [55] & $\star$ & $\star$ & $(5.29,2.32)$ \\
TLSP [56] & $\star$ & $\star$ & $(5,26,2.32)$ \\
\hline
\end{tabular}

$\dagger$ In each binary array $(\bullet, \bullet)$, the first number stands for the achieved RMSE of the corresponding localization method, and the second number denotes the corresponding CRLB.

* This symbol denotes the corresponding localization method is not included in the simulation associated with the corresponding measurement data.

\subsection{Localization Accuracy Comparison}

In this part, the proposed VIP algorithm is compared with several existing localization methods, e.g., ISP [20], ESP [21], PSO [51], WLSP [52], SOCP [53], SDP [54], ASA [55] and TLSP [56] in Scenario C, where RSS, TOA and AOA data are considered, to demonstrate its localization accuracy.

The simulation settings are summarized in TABLE 2. The localization methods corresponding to different measurement choices are introduced in TABLE 3 . The achieved RMSEs of different localization methods with various measurement data are presented in TABLE 5.

As shown in TABLE 5, the proposed VIP scheme outperforms the localization methods mentioned above in all RSS, TOA and AOA cases due to its ability to exploit (1) the underlying statistics of random measurement accuracy \& inaccurate node locations and (11) the global information inside network measurements \& prior location information.

In addition, each of the ISP, PSO and EMP methods fails to reach the CRLB, due to the limited representation efficiency of particles. In contrast, the VIP method can iteratively improve the representation efficiency of variational particles to reduce the approximation error, by exploiting the further knowledge via variational information exchange among nondeterministic variables (e.g., $\left\{\mathbf{s}_{i}, \mathbf{s}_{j}, \mathrm{w}_{i, j} \mid \forall j \in \Psi_{i}\right\}$ ).

The other methods like WLSP, SOCP, SDP, ASA, TLSP also fail to reach the CRLB, and there is localization performance loss compared with the proposed VIP method in all RSS, TOA and AOA cases, due to (1) the lack of mechanism to capture the random measurement accuracy \& reference node uncertainty, and (11) the failure in exploiting global knowledge inside priori location information $\left\{\boldsymbol{\mu}_{i}, \mathbf{U}_{i} \mid \forall i=\right.$
$1: M\}$ and measurement data $\left\{\mathrm{z}_{i, j} \mid \forall j \in \Psi_{i}, \forall i=1: M\right\}$.

\section{Practical Experiment}

\subsection{Experiment Setup}

In order to capture the statistical distribution of realistic measurement precision $\mathrm{w}_{i, j}$ in dynamic environments and to verify the VIP algorithm, we conduct a practical experiment in a static WSN.

The experiment area is a $9[\mathrm{~m}] \times 6[\mathrm{~m}] \times 2.8[\mathrm{~m}]$ (indoor) lab at Southwest Jiaotong University, which is illustrated in Fig. 9(a). We only consider the RSS measurement in this experiment, and the conclusions in the following can be easily extended to the TOA and AOA-based localization. Each sensor node is equipped with an IEEE 802.15.4 ZigBee Module operating at $2.4 \mathrm{GHz}$. A prototype of sensor node is presented in Fig. 9(b). The sensing range of each sensor node is about $r_{s}=20[\mathrm{~m}]$ in an indoor environment.

All RSS data between distinct sensor nodes are collected and read out by a gateway connected to a laptop computer, as shown in Fig. 9(a). The deployment area of this experiment is crowded with students with normal walking speed, which is consistent with a typical dynamic situation in shopping mall and central business district.

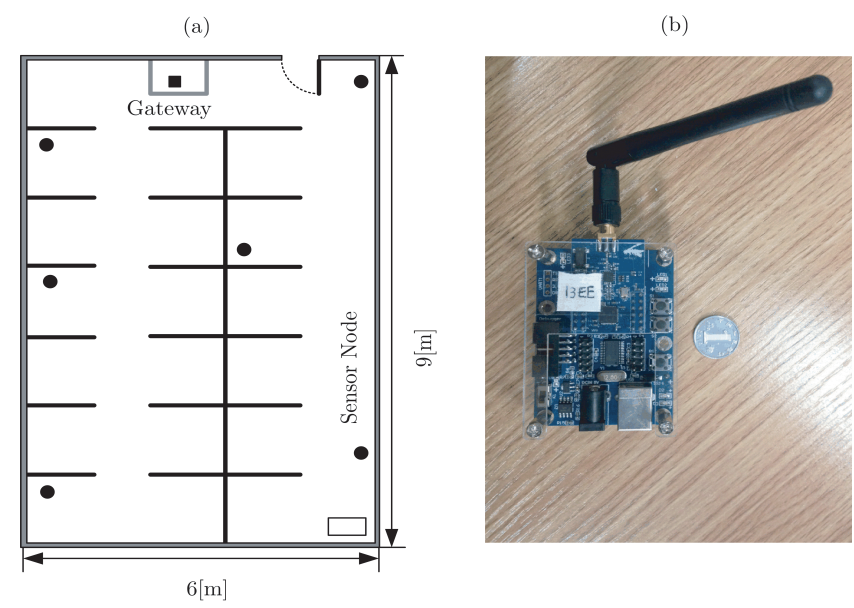

Fig. 9. An illustration of the WSN layout and a prototype of sensor node employed in the practical experiments.

\subsection{Wishart Priori Model}

In order to verify the Wishart hyperpriori model of measurement precision $\mathrm{w}_{i, j}$ assumed in Eq. (7), in this part, a realistic experiment in aforementioned dynamic indoor environment with random walking students is performed. In this experiment, for each node pair $\left(\mathbf{s}_{i}, \mathbf{s}_{j}\right)$, a total of $3 \times 10^{4}$ RSS measurements $\mathrm{z}_{i, j}$ are collected to calculate the 
measurement precision $\mathrm{w}_{i, j}$, and a total of $10^{5}$ measurement precision $\mathrm{w}_{i, j}$ from different node pairs are calculated to count its statistical distribution. ${ }^{7}$
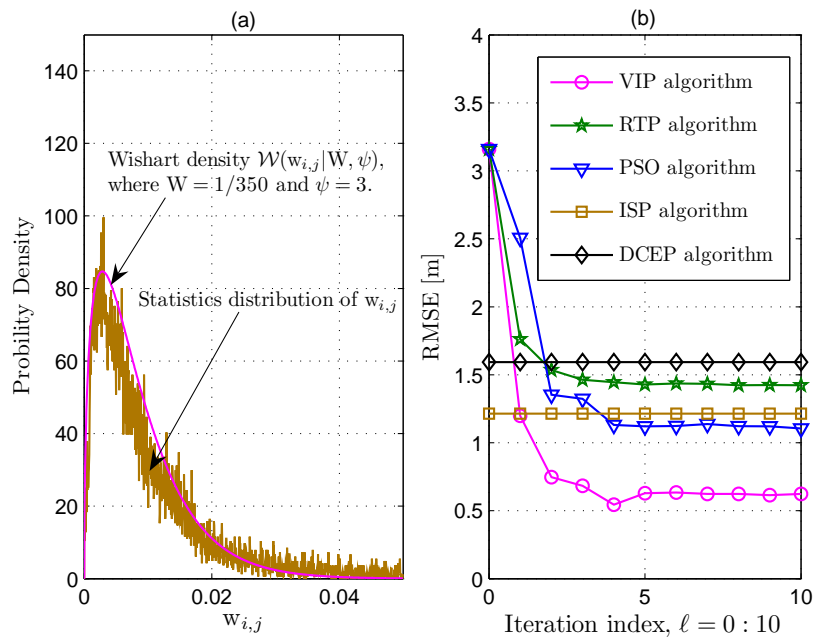

Fig. 10. Statistical probability distribution of measurement precision $\mathrm{w}_{i, j}$ (subfigure (a)) and the resulting localization errors (subfigure (b)).

The associated probability density function of RSS measurement precision $\mathrm{w}_{i, j}$ is shown in Fig. 10(a). We can see that the RSS measurement precisions $\mathrm{w}_{i, j}$ of different sensor node pairs can be approximately described by a Wishart distribution of $\psi=3$ and $\mathrm{W}=1 / 350$. The randomness of $\mathrm{w}_{i, j}$ stems mainly from four reasons: (1) different situations of the measurement links for different node pairs $\left(\mathbf{s}_{i}, \mathbf{s}_{j}\right)$, (11) dynamic wireless environments, (111) different hardware condition leading to different strength of thermal noises, and (iv) different device orientation.

We can also notice that, there still exists a small gap between the statistical distribution and the matched Wishart density. Based on the Large Number Law and Central Limit Theorem, this gap will be dismissed when the measurement sampling size is sufficiently large.

\subsection{Practical Localization Errors}

We compared the proposed VIP scheme with other localization methods, such as ISP algorithm [20], mulihop-aided PSO method [51], rigid transformation-based positioning (RTP) [57] and dynamic circle expanding-based positioning (DCEP) [58], to reveal the VIP-based localization performance against these typical algorithms in the same scenario.

As shown in Fig. 10(b), the VIP-based localization outperforms other algorithms, due to the benefit from variational message propagation and harnessing of the nondeterministic measurement precision. The ISP algorithm loses localization accuracy since it fails to exploit posteriori information of reference node locations $\left\{\mathbf{s}_{j}: \forall j \in \Psi_{i}\right\}$ and the nondeterministic measurement precision $\left\{\mathrm{w}_{i, j}: \forall j \in \Psi_{i}\right\}$, and so are other methods such as RTP and PSO. In addition, since the RTP method is a statistical-independent algorithm, it is very sensitive to the measurement noise intensity. The same problem remains in the DCEP method. Also, since the

\footnotetext{
${ }^{7}$ We employ $\mathrm{w}_{i, j}=\mathbb{E}\left\{\left(\mathrm{z}_{i, j}-\overline{\mathrm{z}}_{i, j}\right)^{-2}\right\}$ to count the statistical measurement precision in experiments, where $\bar{z}_{i, j}$ denotes the average.
}

RTP method highly depends on the rigid geometry among network nodes, it works well in a dense network.

In addition, when the objective function is seriously nonconvex owing to shadowing and nonlinear measurement function, the final solution of PSO is not sufficiently stable, which requires a large amount of searching particles. In particular, the PSO algorithm is highly dependent on the initial particle deployment and the weighting strategy of its particles, thus an optimal weighting method based on the statistical knowledge is necessary, as in the VIP algorithm. On the other hand, the VIP algorithm requires a proper importance sampling function to improve its representation efficiency of particles.

\section{Concluding Remarks}

In this paper, we investigate the cooperative network localization in WSNs, in the presence of sensor node location errors and nondeterministic measurement accuracy. We exploit the statistical information of these two nondeterministic factors to develop a mean-field VIP scheme to localize all network nodes in parallel. The proposed VIP algorithm can reap more potentials not only from the global localization information propagation among network nodes, but also from the variational message sharing among the MB variables.

In addition, a concise CRLB of the VIP scheme is derived to unveil the impact of those two nondeterministic factors on the achievable localization performance. In particular, it discloses that, the priori information $\mathbf{U}_{i}$, the measurement precision $\eta$, the measurement resolution $\mathbf{A}_{i, j}$ and the reference location precisions $\mathbf{U}_{j}$ propagate like the Ohm's Law in circuit theory (see Fig. 4). It is shown that the degradation owing to either the measurement uncertainty or the reference node location uncertainty can effectively be reduced through the global network node localization cooperation with more network measurements. The proposed VIP scheme and its performance analysis can subsume the traditional localization issue as a special case wherein the measurement precision is deterministic and known. Furthermore, we have the following conclusions.

- When the measurement accuracies of network nodes are nondeterministic, the VIP error bound depends on its expectation only.

- The final localization accuracy scales linearly with the reference cluster size. If the reference cluster size is sufficiently large, the localization error can be made arbitrarily small in theory.

- Incorporating one more node with inaccurate initial location into the associated reference cluster can still statistically improve localization accuracy for an unbiased localization system.

The emphasis of our work in this paper is on the cooperative localization algorithm design in adverse environments with significant uncertainties and on the associated localization error propagation mechanism. In the near future, the pros and cons of different cooperative localization schemes in more practical scenarios will be studied to explicitly address the localization competitions, in terms of the localization accuracy, the implementation complexity, the required signaling basis and other related technical issues. Moreover, 
the cooperative localization in more diverse and dynamical environments, for instance, shopping mall, railway station, underground parking, will be tested in the near future to provide further practical evidence. The presented VIP algorithm is generic and widely applicable, we would like to test the cooperative localization in AOA and TOA scenarios as follow-up work in the future.

\section{ACKNOWLEDGMENTS}

This work was supported by the NSFC (No. 61271246), and was also partly supported by Royal Academy of Engineering Award (batch reference 1314-2). In addition, the authors would like to thank Mr. Zefeng Dong and Mr. Hanbing Huang for conducting the experiments.

\section{REFERENCES}

[1] H. Li, L. Sun, Haojin Zhu, X. Lu and X. Cheng, "Achieving privacy preservation in WiFi fingerprint-based localization". INFOCOM, 2014 Proceedings IEEE, Vol.84, No.1, 2014, pp.2337-2345.

[2] M. Li, H. Zhu, Z. Gao, S. Chen, L. Yu, S. Hu and K. Ren, "All your location are belong to us: breaking mobile social networks for automated user location tracking," MobiHoc, 2014, pp.43-52.

[3] I. F. Akyildiz, W. Su, Y. Sankarasubramaniam and E. Cayirci, "A survey on sensor networks." IEEE Communications magazine, Vol.40, No.8, 2002, pp.102-114.

[4] D. Dardari, A. Conti, C. Buratti and R. Verdone, "Mathematical evaluation of environmental monitoring estimation error through energy-efficient wireless sensor networks." IEEE Trans. Mobile Computing, Vol.6, No.7, 2007, pp.790-802.

[5] Y. Chen, D. Lymberopoulos, J. Liu and B. Priyantha, "Indoor Localization Using FM Signals," IEEE Trans. Mobile Computing, Vol.12, No.8, 2013, pp.1502-1517.

[6] A. Conti, M. Guerra, D. Dardari, N. Decarli. and M. Z. Win, "Network experimentation for cooperative localization," IEEE Journal on Selected Areas in Communications, Vol.30, No.2, 2012, pp.467-475.

[7] W. Ouyang, A.K-S. Wong and C.-T. Lea, "Received Signal Strengthbased Wireless Localization via Semidefinite Programming: Noncooperative and Cooperative schemes." IEEE Trans. Veh. Techn., Vol.59, No.3, 2010, pp.1307-1318.

[8] Y. Shen and M. Z. Win. "Fundamental limits of wideband localization -Part I: A general framework." IEEE Trans. Inform. Theory, Vol.56, No.10, 2010, pp.4956-4980.

[9] D. Dardari, A. Conti, U. Ferner, A. Giorgetti and M. Z. Win, "Ranging with ultrawide bandwidth signals in multipath environments." Proceedings of the IEEE, Vol.97, No.2, 2009, pp.404-426.

[10] S. Bartoletti, W. Dai, A. Conti and M. Z. Win, "A mathematical model for wideband ranging." IEEE Journal of Selected Topics in Signal Processing, Vol.9, No.2, 2015, pp.216-228.

[11] Y. Shen, H. Wymeersch and M. Z. Win, "Fundamental limits of wideband localization-Part II: Cooperative Networks," IEEE Trans. Inform. Theory, Vol.56, No.10, 2010, pp.4956-4979.

[12] X. Jun, M. Ma and C. L. Law, "Cooperative angle-of-arrival position localization," Measurement, Vol.59, 2015, pp.302-313.

[13] Y. Dodge and D. R. Cox. "The Oxford dictionary of statistical terms." Oxford University Press, Vol.167, No.2, 2004, pp. 377-377.

[14] K. O. Arras, N. Tomatis, B. T. Jensen and R. Siegwart, "Multisensor on-the-fly localization: - Precision and reliability for applications." Robotics \& Autonomous Systems, Vol.34, No.2-3, 2001, pp.131-143.

[15] R. Feng, X. Guo, N. Yu and J. Wan, "Robust Multihop Localization for Wireless Sensor Networks with Unreliable Beacons," International Journal of Distributed Sensor Networks, Vol.8, No,6, 2012.

[16] K. Shrawan and D. K. Lobiyal, "An advanced dv-hop localization algorithm for wireless sensor networks," Wireless Personal Communications, Vol.71, No.2, 2013, pp.1365-1385.

[17] L. Wang, T. K. Hon, J. D. Reiss and A. Cavalaro, "Self-localization of Ad-Hoc Arrays Using Time Difference of Arrivals", IEEE Transactions on Signal Processing, Vol.64, No.4, 2016, pp.1018-1033.

[18] K. C. Ho, X. N. Lu and L. Kovavisaruch, "Source Localization Using TDOA and FDOA Measurements in the Presence of Receiver Location Errors: Analysis and Solution," IEEE Trans. Signal Processing, Vol.55, No.2, 2007, pp.684-696.
[19] B. Hao, "On the Cramer-Rao Bound of Multiple Sources Localization Using RDOAs and GROAs in the Presence of Sensor Location Uncertainties," Proc. IEEE WCNC, 2012.

[20] M. Vemula, M. F. Bugallo and P. M. Djuric, "Sensor Selflocalization with Beacon Position Uncertainty," Signal Processing, Vol.89, No.6, 2009, pp.1144-1154.

[21] B. Li, N. Wu, H. Wang and J. Kuang, "Nodes localization with inaccurate anchors via EM algorithm in wireless sensor networks," 2014 IEEE International Conference on Communications Workshops (ICC), Sydney, NSW, 2014, pp. 121-126.

[22] D. Roee and L. Lampe, "Underwater localization with timesynchronization and propagation speed uncertainties," IEEE Trans. Mobile Computing, Vol.12, No.7, 2013, pp.1257-1269.

[23] A. Yassin, Y. Nasser, M. Awad, A. A1-Dubai, R. Liu, C. Yuen, R. Raulefs, "Recent Advances in Indoor Localization: A Survey on Theoretical Approaches and Application," IEEE Communications Surveys $\mathcal{E}$ Tutorials, 2016

[24] N. Bulusu, J. Heidemann and D. Estrin, "GPS-less low-cost outdoor localization for very small devices," IEEE Personal Communications, Vol.7, No.5, 2000, pp.28-34.

[25] J. Teng, H. Snoussi , C. Richard and R. Zhou, "Distributed Variational Filtering for Simultaneous Sensor Localization and Target Tracking in Wireless Sensor Networks," IEEE Trans. Veh. Techn., Vol.61, No.5, 2012, pp.2305-2318.

[26] A. Riad and N. Aouf, "Visual information to enhance time difference of arrival based acoustic localization," Proc. of 2014 IEEE International Conference on Aerospace Electronics and Remote Sensing Technology (ICARES), 2014.

[27] G. Giorgetti, S. K. Gupta and G. Manes, "Understanding the limits of RF-based collaborative localization," IEEE/ACM Trans. Networking, Vol.19, No.6, 2011, pp.1638-1651.

[28] W. Yuan, N. Wu, B. Li, H. Wang and J. Kuang, "A low-complexity cooperative localization algorithm based on variational message passing in wireless networks," Proc. of 2014 Sixth International Conference on Wireless Communications and Signal Processing (WCSP), 2014.

[29] K. Yang, G. Wang and Z. Luo, "Efficient convex relaxation methods for robust target localization by a sensor network using time differences of arrivals," IEEE Trans. Signal Processing, Vol.57, No.7, 2009, pp.2775-2784.

[30] H. Wymeersch, J. Lien and M. Z. Win, "Cooperative localization in wireless networks," Proceedings of the IEEE, Vol.97, No.2, 2009, pp.427-450.

[31] I. Sharp, K. Yu and T. Sathyan, "Positional accuracy measurement and error modeling for mobile tracking," IEEE Trans. Mobile Computing, Vol.11, No.6, 2012, pp.1021-1032.

[32] F. Gustafsson and F. Gunnarsson. "Mobile positioning using wireless networks: possibilities and fundamental limitations based on available wireless network measurements." IEEE Signal Processing Magazine, Vol.22, No.4, 2005, pp.41-53.

[33] M. Z. Win, A. Conti, S. Mazuelas, Y. Shen, W. M. Gifford, D. Dardari and M. Chiani, "Network localization and navigation via cooperation," IEEE Communications Magazine, Vol.49, No.5, 2011, pp.56-62.

[34] I. Güvenç, C. C. Chong, F. Watanabe and H. Inamura, "NLOS identification and weighted least-squares localization for UWB systems using multipath channel statistics," EURASIP Journal on Advances in Signal Processing, Vol.2008, No.36, 2008.

[35] I. Güvenç and C. C. Chong. "A survey on TOA based wireless localization and NLOS mitigation techniques," IEEE Communications Surveys \& Tutorials Vol.11, No.3, 2009, pp.107-124.

[36] P. M. Djuric, M. V. Vemula, M. R. Bugallo and J. Miguez, "Noncooperative Localization of Binary Sensors," Proc. of IEEE 13th Workshop on Statistical Signal Processing, 2005.

[37] A. Goldsmith, Wireless Communications, Cambridge University Press, UK, 2005.

[38] C. M. Bishop "Pattern recognition and machine learning." Company New York Ny, Vol.16, No.4, 2006, pp.104-155.

[39] F. Li, R. Fergus and P. Perona. "One-shot learning of object categories," IEEE Trans. Pattern Analysis and Machine Intelligence, Vol.28, No.4, 2006, pp.594-611.

[40] K. P. Murphy, "Conjugate Bayesian analysis of the Gaussian distribution." Technical Report, 2007.

[41] H. Snoussi and C. Richard, Ensemble learning online filtering in wireless sensor networks, Proc. IEEE Int. Conf. Commun. Syst., 2006, pp. 1-5. 
[42] J. Teng, H. Snoussi, and C. Richard, Decentralized variational filtering for target tracking in binary sensor networks, IEEE Trans. Mobile Computing, Vol. 9, No. 10, pp. 1465-1477, Oct. 2010.

[43] T. Y. Chow, "What is a closed-Form Number?." American Mathematical Monthly, Vol.106, No.5, 1999, pp.440-448.

[44] C. W. Fox and S. J. Roberts, "A Tutorial on Variational Bayesian Inference," Artificial Intelligence Review, Vol.38, No.2, 2012, pp.85-95.

[45] M. S. Arulampalam, S. Maskell, N. Gordon and T. Clapp," A tutorial on particle filters for online nonlinear/non-Gaussian Bayesian tracking," IEEE Trans. Signal Processing, Vol.50, No.2, 2002, pp.174188.

[46] G. Wang and K. Yang, "A new approach to sensor node localization using RSS measurements in wireless sensor networks," IEEE Trans. Wireless Communications, Vol.10, No.5, 2011, pp.1389-1395.

[47] K. Friston, J. Mattout, N. Trujillo-Barreto, J. Ashburner and W. Pennya, "Variational free energy and the Laplace approximation," NeuroImage, ELSEVIER, Vol.34, No.1, 2006, pp.220-234.

[48] C. F. Aliferis, A. Statnikov, T. Tsamardinos, S. Mani, and X. D. Koutsoukos, "Local causal and markov blanket induction for causal discovery and feature selection for classification part I: Algorithms and empirical evaluation," J. Mach. Learn. Res., Vol.11, 2010, pp.171234.

[49] B. Wang and D. M. Titterington, "Convergence and asymptotic normality of variational Bayesian approximations for exponential family models with missing values," Proc. of the 20th Conference on Uncertainty in Artificial Intelligence, AUAI Press, 2004.

[50] S. M. Kay, Fundamentals of Statistical Signal Processing, Vol. 2: Detection theory. Prentice Hall PTR, 1998

[51] R. V. Kulkarni and G. K. Venayagamoorthy, "Particle swarm optimization in wireless-sensor networks: A brief survey." IEEE Trans. Systems, Man, and Cybernetics, Part C: Applications and Reviews, Vol.41, No.2, 2011, pp.262-267.

[52] H. EI-Sayed, G. Athanasiou, and C. Fischione. "Evaluation of Localization Methods in Millimeter-Wave Wireless Systems." IEEE International Workshop on Computer Aided Modeling and Design of Communication Links and Networks, IEEE, 2014, pp.345-349.

[53] S. Tomic, M. Beko, R. Dinis, and V. Lipovac, "Efficient Estimator for Distributed RSS-based Localization in Wireless Sensor Networks", 11th International Wireless Communications and Mobile Computing Conference, Dubrovnik, Croatia, August 24-28, 2015.

[54] Y. Zou, and Q. Wan, "Asynchrous Time-of-Arrival-based Source Localization with Sensor Position Uncertainties", IEEE Communications Letters, Vol.20, No.9, 2016, pp.1860-1863.

[55] O. Jean, and A. J. Weiss, "Geolocalization by Direction of Arrival Using Arrays With Unknown Orientation." IEEE Transactions on Signal Processing, Vol.62, No.12, June 15, 2014, pp.3135-3142.

[56] H. J. Shao, X. P. Zhang, and Z. Wang, "Efficient Closed-Form Algorithms for AOA Based Self-Localization of Sensor Nodes Using Auxiliary Variables," IEEE Transactions on Signal Processing, Vol.62, No.10, pp.2580-2594, May 15, 2014.

[57] L. Zhang, L. Liu, C. Gotsman and S. J. Gortler, An as-rigidas-possible approach to sensor network localization, ACM Trans. Sensor Networks, Vol.6, No.4, 2010, pp.53-56.

[58] J. A. Jiang, X. Y. Zheng, Y. F. Chen, C. H. Wang, P. T. Chen, C. L. Chuang and C. P. Chen, "A distributed RSS-based localization using a dynamic circle expanding mechanism," IEEE Sensors Journal, Vol.13, No.10, 2013, pp.3754-3766.

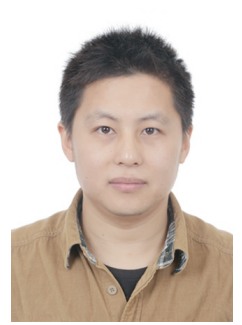

Bingpeng Zhou (S'16-M'17) received the B.Eng degree in electronic and information engineering from Zhongyuan University of Technology, Zhengzhou, P.R. China, in 2010. He is working toward the Ph.D. degree since 2011, with the School of Information Science and Technology, Southwest Jiaotong University, Chengdu, P.R. China, and currently he is a Ph.D. candidate. He is a Visiting Scholar with the Dept. of Electronic and Computer Engineering, Hong Kong University of Science and Technology, Hong Kong, P.R. China, from December 1, 2016. He was a Visiting Ph.D. Student at the 5G Innovation Centre, University of Surrey, Guildford, U.K., from September to November, 2015. His current research interests include wireless localization \& tracking, distribution Bayesian signal processing and rapid-time-varying wireless communication systems.

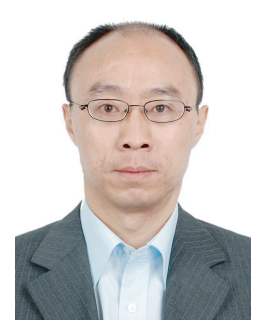

Qingchun Chen (SM'14) received his B.Sc degree and M.Sc degree with honor from Chongqing University, P.R. China, in 1994 and 1997, respectively. He received his Ph.D. degree from Southwest Jiaotong University, P.R. China in 2004. He joined Southwest Jiaotong University since 2004 as an associate professor and then as a full professor since 2009. Dr. Qingchun Chen has authored and coauthored over 90 research papers, two book chapters and 30 patents. Dr. Chen received the 2016 IEEE GLOBECOM Best Paper Award. He served as Associate Editor for IEEE ACCESS (2016-present). His research interest includes wireless communication, wireless network, information coding and signal processing.

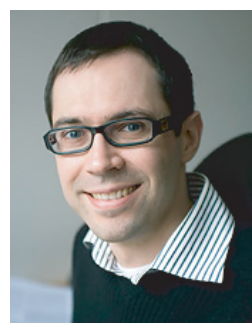

Henk Wymeersch (S'99, M'05) received the $\mathrm{Ph} . \mathrm{D}$. degree in Electrical Engineering/Applied Sciences in 2005 from Ghent University, Belgium. He is currently an Associate Professor with the Department of Signals and System$s$ at Chalmers University of Technology, Sweden. Prior to joining Chalmers, he was a Postdoctoral Associate with the Laboratory for Information and Decision Systems (LIDS) at the Massachusetts Institute of Technology (MIT). He served as Associate Editor for IEEE COMMUNICATION LETTERS (2009-2013), IEEE TRANSACTIONS ON WIRELESS COMMUNICATIONS (2013-present), and the TRANSACTIONS ON EMERGING TELECOMMUNICATIONS TECHNOLOGIES (2011-present).

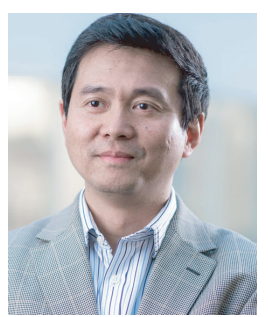

Pei Xiao (SM'11) received the B.Eng, MSc and Ph.D. degree from Huazhong University of Science \& Technology, Tampere University of Technology, Chalmers University of Technology, respectively. Prior to joining the University of Surrey in 2011, he worked as a research fellow at Queen's University Belfast and had held positions at Nokia Networks in Finland. $\mathrm{He}$ is a Reader at University of Surrey and also the technical manager of $5 \mathrm{G}$ Innovation Centre (5GIC), leading and coordinating research activities in all the work areas in 5GIC (http://www.surrey.ac.uk/5gic/what-5g). Dr. Xiao's research interests and expertise span a wide range of areas in communications theory and signal processing for wireless communications.

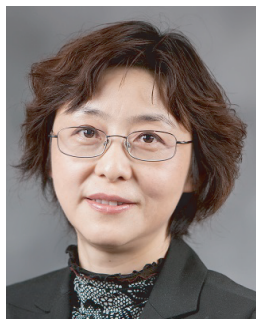

Lian Zhao (S'99-M'03-SM'06) received the Ph.D. degree from the Department of Electrical and Computer Engineering (ELCE), University of Waterloo, Canada, in 2002. She joined the Electrical and Computer Engineering Department Ryerson University, Toronto, Canada, in 2003 and a Professor in 2014. Her research interests are in the areas of wireless communications, radio resource management, power control, cognitive radio and cooperative communications, optimization for complicated systems.

She received the Best Land Transportation Paper Award from IEEE Vehicular Technology Society in 2016; Top 15 Editor in 2015 for IEEE Transaction on Vehicular Technology; Best Paper Award from the 2013 International Conference on Wireless Communications and Signal Processing (WCSP) and Best Student Paper Award (with her student) from Chinacom in 2011; the Ryerson Faculty Merit Award in 2005 and 2007; the Canada Foundation for Innovation (CFI) New Opportunity Research Award in 2005, and Early Tenure and promotion to Associate Professor in 2006. She has been an Editor for IEEE TRANSACTIONS ON VEHICULAR TECHNOLOGY since 2013; workshop co-chair for IEEE/CIC ICCC 2015; local arrangement co-chair for IEEE Infocom 2014; cochair for IEEE Global Communications Conference (GLOBECOM) 2013 Communication Theory Symposium.

She served as an Associate Chair at the Department of Electrical and Computer Engineering at Ryerson University 2013-2015; and a committee member for NSERC (Natural Science and Engineering Research Council of Canada) Discovery Grants Evaluation Group for Electrical and Computer Engineering since 2015. She is a licensed Professional Engineer in the Province of Ontario, a senior member of the IEEE Communication and Vehicular Society. 